\title{
Large sets at infinity and Maximum Principle on unbounded domains for a class of sub-elliptic operators
}

\author{
Stefano Biagi and Ermanno Lanconelli
}

August 28, 2019

\begin{abstract}
Maximum Principles on unbounded domains play a crucial rôle in several problems related to linear second-order PDEs of elliptic and parabolic type. In this paper we consider a class of sub-elliptic operators $\mathcal{L}$ in $\mathbb{R}^{N}$ and we establish some criteria for an unbounded open set to be a Maximum Principle set for $\mathcal{L}$. We extend some classical results related to the Laplacian (by Deny, Hayman and Kennedy) and to the sub-Laplacians on stratified Lie groups (by Bonfiglioli and the second-named author).
\end{abstract}

\section{Introduction and main results}

It is quite well-known that maximum principles on unbounded domains play a crucial rôle in looking for symmetry properties of solutions to semilinear Poissontype equations, by using the celebrated moving planes or sliding method: see, e.g., [5, 6, 7] for the Euclidean setting and see [10, 11 for the Heisenberg group setting.

In the present paper we extend to a wide class of subelliptic PDEs some maximum principles in unbounded domains holding true for the Euclidean Laplace operator (by Deny, Hayman and Kennedy) and for the sub-Laplace operators on stratified Lie groups (by Bonfiglioli and the second-named author).

To be more prices, throughout the sequel we shall be concerned with second-order linear partial differential operators (PDOs, in the sequel) of the form

$$
\mathcal{L}=\frac{1}{V(x)} \sum_{i=1}^{N} \frac{\partial}{\partial x_{i}}\left(\sum_{j=1}^{N} V(x) a_{i, j}(x) \frac{\partial}{\partial x_{j}}\right)=\frac{1}{V(x)} \operatorname{div}(V(x) A(x) \cdot \nabla) .
$$

We shall always assume, without any further comment, that the following structural assumptions are satisfied:

(H1): $V, a_{i, j} \in C^{\infty}\left(\mathbb{R}^{N}, \mathbb{R}\right)$ for all $i, j$ and $V>0$ on the whole of $\mathbb{R}^{N}$;

(H2): the matrix $A(x)=\left(a_{i, j}(x)\right)_{i, j}$ is symmetric and positive semi-definite for every $x \in \mathbb{R}^{N}$. Furthermore,

$$
\operatorname{trace}(A(x))>0 \text { for every } x \in \mathbb{R}^{N} \text {; }
$$


(H3): there exists a real $\varepsilon>0$ such that both $\mathcal{L}$ and $\mathcal{L}_{\varepsilon}:=\mathcal{L}-\varepsilon$ are $C^{\infty}$-hypoelliptic in every open subset 1 of $\mathbb{R}^{N}$.

Under these assumptions, a satisfactory Potential Theory for $\mathcal{L}$ can be constructed (see, e.g., [3, 4]). In this theory, the "harmonic" functions are the $\mathcal{L}$-harmonic functions, that is, the (smooth) solutions to

$$
\mathcal{L} u=0
$$

on some open subset of $\mathbb{R}^{N}$. The corresponding $\mathcal{L}$-subharmonic functions are the upper semi-continuous (u.s.c., for short) functions $u: \Omega \rightarrow[-\infty, \infty$ ) (where $\Omega$ is an open subset of $\mathbb{R}^{N}$ ) such that

(i) $\{x \in \Omega: u(x)>-\infty\}$ is dense in $\Omega$;

(ii) for every bounded open set $V \subseteq \bar{V} \subseteq \Omega$ and for every function $h \mathcal{L}$-harmonic in $V$ and continuous up to $\partial V$ such that $u_{\left.\right|_{\partial V}} \leq h_{\left.\right|_{\partial V}}$, one has $u \leq h$ in $V$.

As a consequence of the (strong) Harnack inequality for $\mathcal{L}$ proved in [4] (and of the fact that $h \equiv 1$ is $\mathcal{L}$-harmonic), the following Maximum Principle for $\mathcal{L}$ subharmonic functions holds true (see Theorem A.2 in the Appendix):

Let $\Omega \subseteq \mathbb{R}^{N}$ be open and bounded and let $u \in \underline{\mathcal{L}}(\Omega)$. Then

$$
\limsup _{x \rightarrow \xi} u(x) \leq 0 \text { for every } \xi \in \partial \Omega \quad \Longrightarrow \quad u \leq 0 \text { in } \Omega .
$$

Here and in what follows, we adopt the subsequent notations:

- $\underline{\mathcal{L}}(\Omega)$ denotes the cone of the $\mathcal{L}$-subharmonic functions in the open set $\Omega \subseteq \mathbb{R}^{N}$;

- $\underline{\mathcal{L}}_{b}(\Omega)$ denotes the cone of the bounded above $\mathcal{L}$-subharmonic functions in $\Omega$;

- $\mathcal{L}(\Omega)$ denotes linear space of the $\mathcal{L}$-harmonic functions in $\Omega$;

- $\overline{\mathcal{L}}(\Omega)$ denotes the cone of the $\mathcal{L}$-superharmonic functions in $\Omega$ (by definition, a function $u$ is $\mathcal{L}$-superharmonic (in $\Omega$ ) if $-u$ is $\mathcal{L}$-subharmonic in the same set).

A simple yet remarkable consequence of (1.2) is the fact that a function $u$ in $C^{2}(\Omega, \mathbb{R})$ is $\mathcal{L}$-subharmonic in $\Omega$ if and only if $\mathcal{L} u \geq 0$ on $\Omega$ (see, e.g., [3]).

Obviously, we cannot expect that the previous Maximum Principle holds true if $\Omega$ is not bounded, and if we do not assume in (1.2) some extra conditions on the function $u$; the main aim of this paper is to provide conditions on an unbounded open set $\Omega$ ensuring (1.2) for every bounded above $\mathcal{L}$-subharmonic function in $\Omega$.

To present our main results, it is convenient to fix the following definition.

Definition 1.1. Let $\Omega \subseteq \mathbb{R}^{N}$ be open. We say that $\Omega$ is a maximum principle set (MP set, in short) for $\mathcal{L}$ if it satisfies the following property:

$$
\left\{\begin{array}{l}
u \in \underline{\mathcal{L}}_{b}(\Omega) \\
\limsup _{x \rightarrow \xi} u(x) \leq 0 \quad \text { for every } \xi \in \partial \Omega \quad \Longrightarrow \quad u \leq 0 \quad \text { in } \Omega .
\end{array}\right.
$$

\footnotetext{
${ }^{1}$ We remind that a linear PDO $P$ with smooth coefficients is $C^{\infty}$-hypoelliptic in an open set $\Omega \subseteq \mathbb{R}^{N}$ if any distributional solution to $P u=f$ is smooth in $\Omega$ whenever $f$ is smooth.
} 
We point out that any u.s.c. function $u: \Omega \rightarrow[-\infty, \infty)$ satisfying the boundary condition in (1.3) is bounded above if $\Omega$ is bounded. Then, by the previously recalled Maximum Principle, every bounded open set is a MP set for $\mathcal{L}$ and in (1.1) we can replace $\underline{\mathcal{L}}_{b}(\Omega)$ with $\underline{\mathcal{L}}(\Omega)$.

As we shall see, the notion of maximum principle set (for $\mathcal{L}$ ) is closely related to the one of $\mathcal{L}$-largeness at infinity, defined as follows.

Definition 1.2. We say that a subset $F$ of $\mathbb{R}^{N}$ is $\mathcal{L}$-thin at infinity if it is possible to find a function $u \in \underline{\mathcal{L}}_{b}\left(\mathbb{R}^{N}\right)$ such that 2

$$
\limsup _{\substack{x \rightarrow \infty \\ x \in F}} u(x)<\limsup _{\substack{x \rightarrow \infty \\ x \in \mathbb{R}^{N}}} u(x) .
$$

If $F \subseteq \mathbb{R}^{N}$ is not $\mathcal{L}$-thin at infinity, we shall say that $F$ is $\mathcal{L}$-large at infinity. Explicitly, $F$ is $\mathcal{L}$-large at infinity if and only if

$$
\limsup _{\substack{x \rightarrow \infty \\ x \in F}} u(x)=\limsup _{\substack{x \rightarrow \infty \\ x \in \mathbb{R}^{N}}} u(x) \quad \text { for every } u \in \underline{\mathcal{L}}_{b}\left(\mathbb{R}^{N}\right) .
$$

Here is our first basic result.

Theorem 1.3. An open set $\Omega \subseteq \mathbb{R}^{N}$ is a maximum principle set for $\mathcal{L}$ if and only if its complement $\mathbb{R}^{N} \backslash \Omega$ is $\mathcal{L}$-large at infinity.

The proof of this theorem will be given in Section 2, In Section 3, assuming that $\mathcal{L}$ is endowed with a global fundamental solution

$$
(x, y) \mapsto \Gamma(x ; y),
$$

smooth out of the diagonal of $\mathbb{R}^{N} \times \mathbb{R}^{N}$ and satisfying suitable structural conditions (see, precisely, assumptions (FS), (G) and (L)), we shall provide a geometrical criterion for a set to be $\mathcal{L}$-large at infinity. This criterion involves the superlevel sets of $\Gamma$, which shall be called $\Gamma$-balls: more precisely, for every $x \in \mathbb{R}^{N}$ and every $r>0$, the $\Gamma$-ball with center at $x$ and radius $r$ is the set

$$
\Omega(x, r):=\left\{y \in \mathbb{R}^{N}: \Gamma(x ; y)>\frac{1}{r}\right\} .
$$

From our structural assumptions on $\Gamma$ it easily follows that the function

$$
\gamma(x, y):= \begin{cases}0, & \text { if } x=y \\ 1 / \Gamma(x ; y), & \text { if } x \neq y\end{cases}
$$

is a pseudo-metric in $\mathbb{R}^{N}$ and that $\Omega(x, r)$ is actually the metric $\gamma$-ball centered at $x$ and with radius $r$, that is,

$$
\Omega(x, r)=\left\{y \in \mathbb{R}^{N}: \gamma(x, y)<r\right\} .
$$

With the $\Gamma$-balls at hand, we can introduce the definition of $p_{\mathcal{L}}$-unbounded set.

\footnotetext{
${ }^{2}$ If $F$ is bounded, we agree to let $\limsup _{\substack{x \rightarrow \infty \\ x \in F}} u(x)=\infty$.
} 
Definition 1.4. Let $F \subseteq \mathbb{R}^{N}$ be any set and let $p \in(1, \infty)$. We say that $F$ is $p_{\mathcal{L}}$-bounded if there exists a countable family $\mathcal{F}=\left\{\Omega\left(x_{n}, r_{n}\right)\right\}_{n \in J}$ such that

(a) $F \subseteq \bigcup_{n \in J} \Omega\left(x_{n}, r_{n}\right)$;

(b) $\sum_{n \in J}\left(\Gamma\left(0 ; x_{n}\right) r_{n}\right)^{p}=\sum_{n \in J}\left(r_{n} / \gamma\left(0, x_{n}\right)\right)^{p}<\infty$.

If $F \subseteq \mathbb{R}^{N}$ is not $p_{\mathcal{L}}$-bounded, we shall say that $F$ is $p_{\mathcal{L}}$-unbounded.

Then we have the following result.

Theorem 1.5. Let $F \subseteq \mathbb{R}^{N}$ be any (non-void) set. If there exists some $p \in(1, \infty)$ such that $F$ is $p_{\mathcal{L}}$-unbounded, then $F$ is $\mathcal{L}$-large at infinity.

The proof of this theorem rests on the following result, which is of independent interest: it shows a deep property of the bounded above $\mathcal{L}$-subharmonic functions.

Theorem 1.6. Let $u \in \underline{\mathcal{L}}_{b}\left(\mathbb{R}^{N}\right)$ and let $p \in(1, \infty)$ be arbitrarily fixed. Then, it is possible to construct a $p_{\mathcal{L}}$-bounded set $F \subseteq \mathbb{R}^{N}$ such that

$$
\lim _{\substack{x \rightarrow \infty \\ x \notin F}} u(x)=\sup _{\mathbb{R}^{N}} u .
$$

In view of the above Theorem 1.5 it seems natural to look for some "simple" criteria allowing to establish if a set $F \subseteq \mathbb{R}^{N}$ is $p_{\mathcal{L}}$-unbounded (for some $p>1$ ). In Section 4 , assuming that the $\Gamma$-balls satisfy a kind of doubling and reverse doubling condition (see, precisely, assumption (D)), we shall obtain such a criterion via the notion of $\Gamma$-cone, which we now introduce.

Definition 1.7. Let $K \subseteq \mathbb{R}^{N}$ be any set. We say that $F$ is $\Gamma$-cone if it contains a countable family $\mathcal{F}=\left\{\Omega\left(z_{j}, R_{j}\right\}_{j \in J}\right.$ of $\Gamma$-balls such that

(i) $\left\|z_{j}\right\| \rightarrow \infty$ as $j \rightarrow \infty$;

(ii) $\liminf _{j \rightarrow \infty} \frac{R_{j}}{\gamma\left(0, z_{j}\right)}>0$.

Then, the following theorem holds true.

Theorem 1.8. Let $F \subseteq \mathbb{R}^{N}$ and let us assume that there exists a $\Gamma$-cone $K \subseteq F$. Then, it is possible to find a real $p>1$ such that $F$ is $p_{\mathcal{L}}$-unbounded.

Gathering together Theorems 1.3, 1.5 and 1.8 we obtain the following result, in which all the hypotheses (H1)-to-(H3), (FS), (G), (L) and (D) are assumed.

Theorem 1.9. The open set $\Omega \subseteq \mathbb{R}^{N}$ is a maximum principle set for $\mathcal{L}$ if one of the following (sufficient) conditions is satisfied:

(i) $\mathbb{R}^{N} \backslash \Omega$ is $\mathcal{L}$-large at infinity (this condition is also necessary);

(ii) $\mathbb{R}^{N} \backslash \Omega$ is $p_{\mathcal{L}}$-unbounded (for a suitable $p>1$ );

(iii) $\mathbb{R}^{N} \backslash \Omega$ contains a $\Gamma$-cone. 
Proof. (i) This is precisely the statement of Theorem 1.3 .

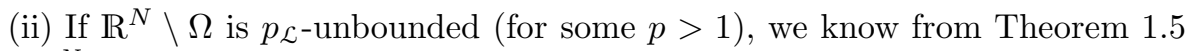
that $\mathbb{R}^{N} \backslash \Omega$ is $\mathcal{L}$-large at infinity; thus, by (i), $\Omega$ is a MP set for $\mathcal{L}$.

(iii) If $\mathbb{R}^{N} \backslash \Omega$ contains a $\Gamma$-cone $K$, we know from Theorem 1.8 that there exists a real $p>1$ such that $\mathbb{R}^{N} \backslash \Omega$ is $p_{\mathcal{L}}$-unbounded; thus, by (ii), we conclude that $\Omega$ is a maximum principle set for $\mathcal{L}$. This ends the proof.

As a consequence of Theorem 1.9 we easily obtain the following result.

Corollary 1.10. Let $\Omega \subseteq \mathbb{R}^{N}$ be an open set satisfying one of conditions (i)-to(iii) in Theorem 1.9. Moreover, let $f: \Omega \times \mathbb{R} \rightarrow \mathbb{R}$ be such that

$$
f(x, z) \leq 0 \quad \text { for every } x \in \Omega \text { and } z \geq 0 .
$$

If $u \in C^{2}(\Omega, \mathbb{R})$ is bounded above and satisfies

$$
\begin{cases}\mathcal{L} u+f(x, u) \geq 0 & \text { in } \Omega \\ \limsup _{x \rightarrow y} u(x) \leq 0 & \text { for every } y \in \partial \Omega\end{cases}
$$

then $u \leq 0$ throughout $\Omega$.

Proof. We argue by contradiction and we assume the existence of some point $x_{0} \in \Omega$ such that $u\left(x_{0}\right)>0$. We then consider the following set

$$
\Omega^{+}:=\{x \in \Omega: u(x)>0\} \neq \varnothing .
$$

By combining (1.6) with (1.7) we infer that, on $\Omega^{+}$, we have $\mathcal{L} u \geq-f(x, u) \geq 0$; as a consequence, $u \in \underline{L}\left(\Omega^{+}\right)$. On the other hand, by the boundary condition in (1.7) and the fact that $u \equiv 0$ on $\partial \Omega^{+} \cap \Omega$, it is readily seen that

$$
\limsup _{x \rightarrow y} u(x) \leq 0 \quad \text { for every } y \in \partial \Omega^{+} .
$$

From this, by arguing exactly as in Lemma 2.1, we infer that the function

$$
v: \Omega \rightarrow \mathbb{R}, \quad v(x)=\max \{u(x), 0\}
$$

is $\mathcal{L}$-subharmonic in $\Omega$; furthermore, since $u$ is bounded above in $\Omega$, the same is true of $v$. Taking into account that, by assumption, $\Omega$ is a MP-set for $\mathcal{L}$, we conclude that $v \leq 0$, whence $u \leq 0$, but this is in contradiction with (1.8).

Finally, in Section 5 we shall prove that the Hörmander's operators sums of squares of homogeneous vector fields satisfy all the hypotheses of the above Theorem 1.9. These operators, precisely, are defined as follows.

Let $X=\left\{X_{1}, \ldots, X_{m}\right\}$ be a family of linearly independent smooth vector fields on Euclidean space $\mathbb{R}^{N}$, with $N \geq 3$, satisfying the following properties:

(I) $X_{1}, \ldots, X_{m}$ are $\delta_{\lambda}$-homogeneous of degree 1 with respect to a family of nonisotropic dilations $\left\{\delta_{\lambda}\right\}_{\lambda>0}$ of the following type

$$
\delta_{\lambda}: \mathbb{R}^{N} \rightarrow \mathbb{R}^{N}, \quad \delta_{\lambda}(x)=\left(\lambda^{\sigma_{1}} x_{1}, \ldots, \lambda^{\sigma_{N}} x_{N}\right),
$$

where $1=\sigma_{1} \leq \ldots \leq \sigma_{N}$ are positive integers; 
(II) $X_{1}, \ldots, X_{m}$ satisfy the Hörmander rank condition, i.e.,

$$
\operatorname{dim}\left\{X(x): X \in \operatorname{Lie}\left\{X_{1}, \ldots, X_{m}\right\}\right\}=N \quad \text { for every } x \in \mathbb{R}^{N} .
$$

Then, the second-order linear operator $\mathcal{L}$ defined by

$$
\mathcal{L}:=\sum_{j=1}^{m} X_{j}^{2}
$$

will be called a homogeneous Hörmander operator.

We want to point out that the class of the homogeneous Hörmander operators contains, as very particular examples, the sub-Laplace operators on stratified Lie groups and the so-called Grushin-type operators on $\mathbb{R}^{N}$ (with $N \geq 3$ ), together with their generalizations: the $\Delta_{\lambda}$-Laplacians (for $\lambda$ smooth) introduced in [19].

When $\mathcal{L}$ is a homogeneous Hörmander operator, our geometrical criteria for $\mathcal{L}$-largeness at infinity $/ p_{\mathcal{L}}$-unboundedness take a more explicit form. While we directly refer to Section 5 for the statement and the proof of such ad-hoc criteria, here we only want to present the "homogeneous" version of the cone criterion.

To this end, it is convenient to fix a definition.

Definition 1.11. Let $C \subseteq \mathbb{R}^{N}$ be any set. We say that $C$ is a non-degenerate $\delta_{\lambda}$-cone if it satisfies the following properties:

(i) $\operatorname{int}(F) \neq \varnothing$;

(ii) there exists $\lambda_{0}>0$ such that $\delta_{\lambda}(C) \subseteq C$ for every $\lambda \geq \lambda_{0}$.

Here, $\left\{\delta_{\lambda}\right\}_{\lambda>0}$ denotes the family of (non-isotropic) dilations associated with the vector fields $X_{1}, \ldots, X_{m}$ and appearing in the above assumption (I).

Then we have the following result, which will be proved is Section 5 .

Proposition 1.12. If $F \subseteq \mathbb{R}^{N}$ contains a non-degenerate $\delta_{\lambda}$-cone, then there exists $p>1$ such that $F$ is $p_{\mathcal{L}}$-unbounded. (in the sense of Definition 1.4).

It can be easily proved that every half-space of $\mathbb{R}^{N}$ contains a non-degenerate $\delta_{\lambda}$-cone (see again Section [5); as a consequence, by combining Proposition 1.12 with Theorem 1.9. we readily obtain the subsequent result.

Theorem 1.13. Let $\mathcal{L}$ be a homogeneous Hörmander operator in $\mathbb{R}^{N}$ (with $N \geq 3$ ) and let $\Omega \subseteq \mathbb{R}^{N}$ be an open set satisfying one of the following conditions:

(i) $\mathbb{R}^{N} \backslash \Omega$ contains a non-degenerate $\delta_{\lambda}$-cone;

(ii) $\Omega$ is contained in a half-space 3 of $\mathbb{R}^{N}$.

Then $\Omega$ is a maximum principle for $\mathcal{L}$.

${ }^{3}$ Note that this is equivalent to say that $\mathbb{R}^{N} \backslash \Omega$ contains a half-space. 
Proof. (i) If $\mathbb{R}^{N} \backslash \Omega$ contains a non-degenerate $\delta_{\lambda}$-cone, it follows from Proposition 1.12 that $\mathbb{R}^{N} \backslash \Omega$ is $p_{\mathcal{L}}$-unbounded (for some $p>1$ ); as a consequence, Theorem 1.9.(ii) allows us to conclude that $\Omega$ is a maximum principle set for $\mathcal{L}$.

(ii) If $\Omega$ is contained in a half-space $H$, then $\mathbb{R}^{N} \backslash \Omega$ contains the half-space $H^{\prime}=\mathbb{R}^{N} \backslash H$; since $H^{\prime}$ contains a non-degenerate $\delta_{\lambda}$-cone (see Remark 5.10), we conclude from (i) that $\Omega$ is a maximum principle set for $\mathcal{L}$.

We point out that, in order to prove that any homogeneous Hörmander operator $\mathcal{L}=\sum_{j=1}^{m} X_{j}^{2}$ satisfies all the hypotheses (H1)-to-(H3), (FS), (G), (L) and (D), we make crucial use of global estimates for two objects associated with $\mathcal{L}$ : its global fundamental solution (see Theorem 5.3 ) and the measure of the balls in the Carnot-Carathéodory metric associated with $X_{1}, \ldots, X_{m}$ (see Theorem 5.1).

When $\mathcal{L}$ is the classical Laplacian or the sub-Laplacian on a stratified Lie group, the maximum principle in Corollary [1.10 was proved in [6] and in [12, respectively. This last paper contains a version of Theorems [1.3, 1.5, 1.6] and 1.13 for the sub-Laplacians setting. We point out that Theorem 1.6, in the case of the classical Laplace operator $\Delta$, is a somehow weaker form of a Deny's theorem for $\Delta$-subharmonic functions (see Theorem 3.1 in the monograph [17]).

A short description of the contents of our paper is now in order.

- In Section 2 we study the relationship between the notion of maximum principle set for $\mathcal{L}$ (see Definition 1.1) and the one of $\mathcal{L}$-thinness (and $\mathcal{L}$-largeness) at infinity (see Definition 1.2).

- In Section 3 we make use of the notion of $p_{\mathcal{L}}$-unboundedness (see Definition 1.4) to give a geometrical sufficient condition for a set to be $\mathcal{L}$-large at infinity.

- In Section 4, by means of the notion of $\Gamma$-cone (see Definition 1.7 we prove that a set is $\mathcal{L}$-large at infinity if it contains a $\Gamma$-cone.

- In Section 5 we prove that our theory apply to every homogeneous Hörmander operator; to this end, we show and use some estimates of the fundamental solution of these operators which are of independent interest.

- Finally, in the Appendix we remind some basic results coming from abstract Potential Theory needed for our study.

\section{$2 \quad \mathcal{L}$-thin sets and Maximum Principle}

The main aim of this section is to prove Theorem 1.3 stated in the Introduction. To this end, we need to demonstrate a couple of preliminary results.

Lemma 2.1. Let $\Omega \subseteq \mathbb{R}^{N}$ be open and let $u \in \underline{L}(\Omega)$ be such that

$$
\limsup _{x \rightarrow \xi} u(x) \leq 0 \quad \text { for every } \xi \in \partial \Omega .
$$

Then the function $v: \mathbb{R}^{N} \rightarrow[-\infty, \infty)$ defined by

$$
v(x)= \begin{cases}\max \{u(x), 0\}, & \text { if } x \in \Omega, \\ 0, & \text { if } x \in \mathbb{R}^{N} \backslash \Omega,\end{cases}
$$

is $\mathcal{L}$-subharmonic in $\mathbb{R}^{N}$. 
The proof of Lemma 2.1 requires some basic notions and facts coming from Potential Theory; for this reason, we postpone it to the Appendix.

Lemma 2.2. Let $F \subseteq \mathbb{R}^{N}$ be any (non-void) set and let $u \in \underline{\mathcal{L}}_{b}\left(\mathbb{R}^{N}\right)$. We assume that $u$ is not constant in $\mathbb{R}^{N}$. Then, the following statements are equivalent:

$$
\begin{aligned}
& \text { (i) } \limsup _{\substack{x \rightarrow \infty \\
x \in F}} u(x)=\limsup _{\substack{x \rightarrow \infty \\
x \in \mathbb{R}^{N}}} u(x) \text {; } \\
& \text { (ii) } \sup _{F} u=\sup _{\mathbb{R}^{N}} u .
\end{aligned}
$$

Proof. (i) $\Rightarrow$ (ii). Since, by assumption, $u$ is not constant in $\mathbb{R}^{N}$ and the constant functions are $\mathcal{L}$-harmonic, the Minimum Principle in Theorem A.2 implies that

$$
u(x)<\sup _{\mathbb{R}^{N}} u \quad \text { for every } x \in \mathbb{R}^{N} .
$$

As a consequence, it is easy to recognize that

$$
\sup _{\mathbb{R}^{N}} u=\limsup _{\substack{x \rightarrow \infty \\ x \in \mathbb{R}^{N}}} u(x) .
$$

This last identity allows us to conclude: indeed, if (2.2) holds, we have

$$
\limsup _{\substack{x \rightarrow \infty \\ x \in F}} u(x) \leq \sup _{F} u \leq \sup _{\mathbb{R}^{N}} u=\limsup _{\substack{x \rightarrow \infty \\ x \in \mathbb{R}^{N}}} u(x) \stackrel{(2.2)}{=} \limsup _{\substack{x \rightarrow \infty \\ x \in F}} u(x),
$$

and thus (2.3) is satisfied, as desired.

(ii) $\Rightarrow$ (i). We first claim that, as a consequence of (2.3), one has

$$
\sup _{F \cap B(0, r)} u<\sup _{F \backslash B(0, r)} u \quad \text { for every } r>0
$$

(here, $B(0, r)$ denotes the Euclidean ball of centre 0 and radius $r$ ).

Indeed, let us assume by contradiction that (2.5) does not hold for some $r_{0}>0$. Since $u$ attains its maximum on any compact subset of $\mathbb{R}^{N}$, it is possible to find a suitable point $x_{0} \in \overline{F \cap B\left(0, r_{0}\right)}$ such that

$$
u\left(x_{0}\right)=\sup _{F \cap B\left(0, r_{0}\right)} \geq \sup _{F \backslash B\left(0, r_{0}\right)} u .
$$

Owing to (2.3), this implies that

$$
u\left(x_{0}\right)=\sup _{F} u=\sup _{\mathbb{R}^{N}} u
$$

which is contradiction with (2.4). Now we have established inequality (2.5), we are ready to conclude: indeed, by letting $r \rightarrow \infty$ in the cited (2.5), we get

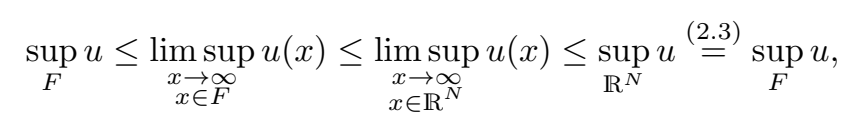

and this proves that (2.2) is satisfied. This ends the proof. 
From Lemma 2.2 and the definition of $\mathcal{L}$-thin set, we obtain the following result.

Corollary 2.3. Let $F \subseteq \mathbb{R}^{N}$ be any set. Then $F$ is $\mathcal{L}$-thin at infinity if and only if it is possible to find a function $u \in \underline{\mathcal{L}}_{b}\left(\mathbb{R}^{N}\right)$ such that

$$
\sup _{F} u<\sup _{\mathbb{R}^{N}} u .
$$

Conversely, $F$ is $\mathcal{L}$-large at infinity if and only if

$$
\sup _{F} u=\sup _{\mathbb{R}^{N}} u \quad \text { for every } u \in \underline{\mathcal{L}}_{b}\left(\mathbb{R}^{N}\right) .
$$

Gathering together Lemmas 2.1 and 2.2, we are ready to prove Theorem 1.3 ,

Proof (of Theorem [1.3). We first prove that, if $\Omega$ is a maximum principle set for $\mathcal{L}$, then its complement $\mathbb{R}^{N} \backslash \Omega$ is $\mathcal{L}$-large at infinity. To this end, we choose $u \in \underline{\mathcal{L}}_{b}(\Omega)$ (which we may assume to be non constant in $\mathbb{R}^{N}$ ) and we let

$$
u_{0}:=\sup _{\mathbb{R}^{N} \backslash \Omega} u
$$

Since $u$ is u.s.c. on $\mathbb{R}^{N}$, for every $\xi \in \partial \Omega$ we have

$$
\limsup _{x \rightarrow \xi} u(x) \leq u(\xi) \leq u_{0} ;
$$

from this, since we are assuming that $\Omega$ is a MP set for $\mathcal{L}$, we obtain

$$
u \leq u_{0} \text { in } \Omega, \quad \text { whence } \sup _{\mathbb{R}^{N}} u=u_{0}=\sup _{\mathbb{R}^{N} \backslash \Omega} u .
$$

By Corollary 2.3, we conclude that $\mathbb{R}^{N} \backslash \Omega$ is $\mathcal{L}$-large at infinity.

We now assume that $\mathbb{R}^{N} \backslash \Omega$ is $\mathcal{L}$-large at infinity and we prove that $\Omega$ is a maximum principle set for $\mathcal{L}$. To this end, we choose once again a function $u \in \underline{\mathcal{L}}_{b}(\Omega)$ (which we may assume to be non constant in $\mathbb{R}^{N}$ ) such that

$$
\limsup _{x \rightarrow \xi} u(x) \leq 0 \quad \text { for every } \xi \in \partial \Omega
$$

and, according to Definition 1.1 we prove that $u \leq 0$ in $\Omega$. To begin with, owing to Lemma 2.1. we see that the function $v: \mathbb{R}^{N} \rightarrow[-\infty, \infty)$ defined by

$$
v(x)= \begin{cases}\max \{u(x), 0\}, & \text { if } x \in \Omega, \\ 0, & \text { if } x \in \mathbb{R}^{N} \backslash \Omega,\end{cases}
$$

is a $\mathcal{L}$-subharmonic function in $\mathbb{R}^{N}$ which is also bounded from above (as the same is true of $u$ ); since we are assuming that the set $\mathbb{R}^{N} \backslash \Omega$ is $\mathcal{L}$-large at infinity, we deduce from Corollary 2.3 that $\sup _{\mathbb{R}^{N}} v=\sup _{\mathbb{R}^{N} \backslash \Omega} v=0$, whence

$$
u(x) \leq \max \{u(x), 0\}=v(x) \leq 0 \text { for every } x \in \Omega .
$$

This ends the proof. 


\section{$3 \quad \mathcal{L}$-thinness at infinity and $p$-boundedness}

The aim of this second section is to demonstrate the geometrical criterion for $\mathcal{L}$ largeness at infinity contained in Theorem 1.5. To this end, as already anticipated in the Introduction, we need to require our PDOs $\mathcal{L}$ to satisfy some additional assumptions, which we now properly introduce.

(FS) First of all, we assume that $\mathcal{L}$ is endowed with a "well-behaved" global fundamental solution, that is, there exists a function

$$
\Gamma: \mathcal{O}:=\left\{(x, y) \in \mathbb{R}^{N} \times \mathbb{R}^{N}: x \neq y\right\} \longrightarrow \mathbb{R}
$$

satisfying the following properties:

(a) $\Gamma \in C^{\infty}(\mathcal{O}, \mathbb{R})$ and $\Gamma(x, y)>0$ for every $x, y \in \mathcal{O}$;

(b) $\Gamma$ is symmetric, that is, $\Gamma(x, y)=\Gamma(y, x)$ for every $(x, y) \in \mathcal{O}$;

(c) for every $x \in \mathbb{R}^{N}$, we have $\Gamma(x, \cdot) \in L_{\text {loc }}^{1}\left(\mathbb{R}^{N}\right)$ and

$$
\int_{\mathbb{R}^{N}} \Gamma(x, y) \mathcal{L} \varphi(y) V(y) \mathrm{d} y=-\varphi(x), \quad \forall \varphi \in C_{0}^{\infty}\left(\mathbb{R}^{N}, \mathbb{R}\right) ;
$$

(d) for every $x \in \mathbb{R}^{N}, \Gamma(x, \cdot)$ has a pole at $x$ and it vanishes at infinity, i.e,

$$
\lim _{y \rightarrow x} \Gamma(x, y)=\infty \quad \text { and } \quad \lim _{\|y\| \rightarrow \infty} \Gamma(x, y)=0 .
$$

For the sake of brevity, given $x \in \mathbb{R}^{N}$, in the sequel we set:

$$
\Gamma_{x}: \mathbb{R}^{N} \backslash\{x\} \longrightarrow \mathbb{R}, \quad \Gamma_{x}(y):=\Gamma(x, y) .
$$

(G) Our second assumption is a sort of "geometric condition" which concerns the super-level sets of the fundamental solution $\Gamma$.

More precisely, for every fixed $x \in \mathbb{R}^{N}$ and every $r>0$, we define the open $\Gamma$-ball of centre $x$ and radius $r$ in the following way

$$
\Omega(x, r):=\left\{y \in \mathbb{R}^{N} \backslash\{x\}: \Gamma_{x}(y)>1 / r\right\} \cup\{x\} ;
$$

we then assume the existence of constant $\theta \in(0,1)$ such that

$$
x \notin \Omega(y, r) \quad \Longrightarrow \quad \Omega(x, \theta r) \cap \Omega(y, \theta r)=\varnothing
$$

for every $x, y \in \mathbb{R}^{N}$ and every $r>0$.

(L) Finally, we suppose that the following Liouville-type theorem holds for $\mathcal{L}$ harmonic functions: if $u \in \mathcal{L}\left(\mathbb{R}^{N}\right)$ is a $\mathcal{L}$-harmonic function which is bounded from above (or from below), then $u$ is constant throughout $\mathbb{R}^{N}$.

Under our assumptions (FS), (G) and (L), we have the following crucial result.

Theorem 3.1. Let $u \in \underline{\mathcal{L}}_{b}\left(\mathbb{R}^{N}\right)$ and let $\mu$ be $\mathcal{L}$-Riesz measure of $u$. Then

$$
\int_{1}^{\infty} \frac{\mu(\Omega(0, r))}{r^{2}} \mathrm{~d} r<\infty .
$$

Moreover, if $u_{0}=\sup _{\mathbb{R}^{N}} u$, we have the representation formula

$$
u(x)=u_{0}-\int_{\mathbb{R}^{N}} \Gamma(x, y) \mathrm{d} \mu(y), \quad \text { for every } x \in \mathbb{R}^{N} .
$$


It is proved in 3 that, if $\Omega \subseteq \mathbb{R}^{N}$ is an open set and $u \in \underline{L}(\Omega)$ (not necessarily bounded above), then $u \in L_{\text {loc }}^{1}(\Omega)$ and $\mathcal{L} u \geq 0$ in the sense of distribution on $\Omega$. Hence, the $\mathcal{L}$-Riesz measure $\mu$ of $u$ is defined by $\mu:=\mathcal{L} u$.

Proof. The proof of (3.4) is analogous to that of [13, Theorem 9.6.1]: it is crucially based on assumption $(\mathrm{G})$ and on the mean value formulas for $\mathcal{L}$ established in $[2$.

As for the proof of (3.5), it can be obtained by combining the Liouville-type theorem in assumption (L) with [3, Remark 5.5] (see also [13, Corollary 9.4.8]).

Remark 3.2. We point out, for future reference, that the "geometric condition" in assumption $(\mathrm{G})$ is actually equivalent to requiring that the function

$$
\mathbb{R}^{N} \times \mathbb{R}^{N} \ni(x, y) \mapsto \gamma(x, y)=\gamma_{x}(y):= \begin{cases}1 / \Gamma(x, y), & \text { if } x \neq y \\ 0, & \text { if } y=x\end{cases}
$$

satisfies a pseudo-triangle inequality, that is, there exists $\mathbf{c}>1$ such that

$$
\gamma(x, y) \leq \mathbf{c}(\gamma(x, z)+\gamma(z, y)) \quad \text { for every } x, y, z \in \mathbb{R}^{N} .
$$

Indeed, if (3.6) holds, it is very easy to recognize that assumption $(G)$ is satisfied with $\theta=1 /(2 \mathbf{c})<1$. On the other hand, if (3.3) holds, one has

$$
\Omega(x, \theta / \Gamma(x, y)) \cap \Omega(y, \theta / \Gamma(x, y))=\varnothing \quad \text { for every } x \neq y .
$$

From this, we easily obtain the validity of (3.6) with $\mathbf{c}=1 / \theta>1$.

Remark 3.3. By Remark 3.2 and the properties of $\Gamma$ listed in assumption (FS), we derive that $\gamma=1 / \Gamma$ is a quasi-distance in $\mathbb{R}^{N}$. In fact, we have

- $\gamma \geq 0$ on $\mathbb{R}^{N} \times \mathbb{R}^{N}$ and $\gamma(x, y)=0$ if and only if $x=y$;

- $\gamma(x, y)=\gamma(y, x)$ for every $x, y \in \mathbb{R}^{N}$;

- $\gamma(x, y) \leq \mathbf{c}(\gamma(x, z)+\gamma(z, y))$ for every $x, y, z \in \mathbb{R}^{N}$.

Furthermore, for every $x \in \mathbb{R}^{N}$ and every $r>0$ we have

$$
\Omega(x, r)=\left\{y \in \mathbb{R}^{N}: \gamma(x, y)<r\right\} .
$$

Now we have introduced assumptions (FS), (G) and (L), we proceed to the proof of Theorem 1.5. To begin with, we list in the next remark some useful properties of $p_{\mathcal{L}}$-bounded sets which follow immediately from Definition 1.4

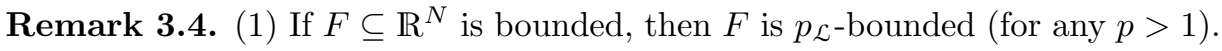

(2) If $F_{0} \subseteq F$ and $F$ is $p$-bounded, then also $F_{0}$ is $p_{\mathcal{L}}$-bounded.

(3) If $\left\{F_{j}\right\}_{j=1}^{n}$ are $p_{\mathcal{L}}$-bounded (for the same $p$ ), then $F=\cup_{j} F_{j}$ is $p_{\mathcal{L}}$-bounded.

(4) If $F_{0} \subseteq F$ is $p_{\mathcal{L}}$-bounded, then $F \backslash F_{0}$ is $p_{\mathcal{L}}$-unbounded whenever $F$ is.

We then turn to demonstrate Theorem 1.6 stated in the Introduction: as anticipated, this result is the key tool for proving Theorem 1.5. In its turn, the proof of the cited Theorem 1.6 is crucially based on the next lemma. 
Lemma 3.5. Let $\mu$ be a positive Radon measure on $\mathbb{R}^{N}$ such that $\mu_{0}=\mu\left(\mathbb{R}^{N}\right)$ is finite. Moreover, let $p \in(1, \infty)$ be fixed and let $h>0$. Then the set

$$
\left\{x \in \mathbb{R}^{N}: \Gamma \mu(x) \geq h\right\}=\left\{x \in \mathbb{R}^{N}: \int_{\mathbb{R}^{N}} \Gamma(x, y) \mathrm{d} \mu(y) \geq h\right\}
$$

can be covered a finite or countable family $\mathcal{F}=\left\{\overline{\Omega\left(x_{n}, r_{n}\right)}\right\}_{n \in J}$ of closed $\Gamma$-balls satisfying the following property: there exists a real constant $A_{p}>0$ such that

$$
\sum_{n \in J}\left(r_{n}\right)^{p}<A_{p}\left(\frac{\mu_{0}}{h}\right)^{p}
$$

Proof. For every fixed natural $n$, we define

$$
r_{n}:=\frac{\mu_{0}}{h} \cdot 2^{-2 n /(p+1)}
$$

and we choose a maximal family $\mathcal{D}_{n}$ of disjoint $\Gamma$-balls of radius $r_{n}$ such that

$$
\mu(B) \geq \frac{\mu_{0}}{2^{n}} \quad \text { for every } B \in \mathcal{D}_{n} .
$$

Since, by assumption, $\mu_{0}=\mu\left(\mathbb{R}^{N}\right)<\infty$ and the $\Gamma$-balls in $\mathcal{D}_{n}$ are disjoint, it is readily seen that $\mathcal{D}_{n}$ consists of at most $k_{n} \leq 2^{n}$ elements; hence, we write

$$
\mathcal{D}_{n}=\left\{\Omega_{k, n}=\Omega\left(x_{k, n}, r_{n}\right): k=1, \ldots, k_{n}\right\} .
$$

If $\theta$ is the constant appearing in assumption $(\mathrm{G})$, we then define

$$
F:=\bigcup_{n=1}^{\infty} \bigcup_{k=1}^{k_{n}} \Omega\left(x_{k, n}, r_{n} / \theta\right)
$$

We now observe that, if $x \notin F$, then $\Omega\left(x, r_{n}\right)$ does not intersect any element of the family $\mathcal{D}_{n}$ : in fact, since $x \notin \Omega\left(x_{k, n}, r_{n} / \theta\right)$, assumption $(\mathrm{G})$ implies that

$$
\Omega\left(x, r_{n}\right) \cap \Omega\left(x_{k, n}, r_{n}\right)=\varnothing \quad \text { for every } n \in \mathbb{N} \text { and every } k \leq k_{n} .
$$

As a consequence, since $\mathcal{D}_{n}$ is maximal, we infer that $\Omega\left(x, r_{n}\right) \notin \mathcal{D}_{n}$, whence

$$
\mu\left(\Omega\left(x, r_{n}\right)\right) \leq \frac{\mu_{0}}{2^{n}} \quad \text { for every } n \in \mathbb{N} .
$$

In particular, $\mu(\{x\})=0$. For every $x \in F$, we then have

$$
\begin{aligned}
\Gamma \mu(x) & =\int_{\mathbb{R}^{N} \backslash\{0\}} \Gamma(x, y) \mathrm{d} \mu(y) \\
& =\left(\int_{\mathbb{R}^{N} \backslash \overline{\Omega\left(x, r_{1}\right)}}+\sum_{n=1}^{\infty} \int_{\Omega\left(x, r_{n}\right) \backslash \overline{\Omega\left(x, r_{n}+1\right)}} \Gamma(x, y) \mathrm{d} \mu(y)\right. \\
& \leq \frac{\mu_{0}}{r_{1}}+\sum_{n=1}^{\infty} \frac{\mu\left(\Omega\left(x, r_{n}\right)\right)}{r_{n+1}} \leq \mu_{0}\left(\frac{1}{r_{1}}+\sum_{n=1}^{\infty} \frac{1}{2^{n} r_{n+1}}\right) \\
& =\mu_{0} \sum_{n=1}^{\infty} 2^{1-n} r_{n}^{-1}=2 h \sum_{n=1}^{\infty}\left(2^{\frac{1-p}{1+p}}\right)^{n} \\
& =A_{p} h,
\end{aligned}
$$


where $A_{p}$ only depends on $p>1$. We have thus proved that, for every $x \in F$, we have $\Gamma \mu(x) \leq A_{p} h$; this obviously implies the inclusion

$$
\left\{x \in \mathbb{R}^{N}: \Gamma \mu(x)>A_{p} h\right\} \subseteq F=\bigcup_{n=1}^{\infty} \bigcup_{k=1}^{k_{n}} \Omega\left(x_{k, n}, r_{n} / \theta\right) .
$$

Furthermore, since $k_{n} \leq 2^{n}$, we have

$$
\begin{aligned}
\sum_{n=1}^{\infty} \sum_{k=1}^{k_{n}}\left(r_{n}\right)^{p} & \leq\left(\frac{\mu_{0}}{h}\right)^{p} \cdot \sum_{n=1}^{\infty} 2^{n}\left(r_{n}\right)^{p} \\
= & \left(\frac{\mu_{0}}{h}\right)^{p} \cdot \sum_{n=1}^{\infty}\left(2^{\frac{1-p}{1+p}}\right)^{n}=A_{p}\left(\frac{\mu_{0}}{h}\right)^{p} .
\end{aligned}
$$

Since the constant $A_{p}$ is positive and only depends on $p$, the lemma is proved.

With Lemma 3.5 at hand, we can prove Theorem 1.6 .

Proof (of Theorem [1.6). Let $u$ be as in the statement of the theorem. Moreover, let $\theta$ be the constant appearing in assumption $(\mathrm{G})$ and let $n \in \mathbb{N}$ be fixed. If $\mu$ is the $\mathcal{L}$-Riesz measure of $u$ and $u_{0}=\sup _{\mathbb{R}^{N}} u$, by Theorem 3.1 we have

$$
u(x)=u_{0}-\int_{\mathbb{R}^{N}} \Gamma(x, y) \mathrm{d} \mu(y)=I_{1}(x)+I_{2}(x)+I_{3}(x),
$$

where we have used the notations

$$
\begin{array}{r}
I_{1}(x):=\int_{\left\{\Gamma_{0}(y) \geq \theta^{n-1}\right\}} \Gamma(x, y) \mathrm{d} \mu(y) ; \\
I_{2}(x):=\int_{\left\{\theta^{n+2}<\Gamma_{0}(y)<\theta^{n-1}\right\}} \Gamma(x, y) \mathrm{d} \mu(y) ; \\
I_{3}(x):=\int_{\left\{\Gamma_{0}(y) \leq \theta^{n+2}\right\}} \Gamma(x, y) \mathrm{d} \mu(y) .
\end{array}
$$

We then consider the set $\Omega_{n}$ defined by

$$
\Omega_{n}:=\left\{x \in \mathbb{R}^{N}: \theta^{n+1}<\Gamma_{0}(x) \leq \theta^{n}\right\} .
$$

and we proceed by estimating $I_{1}(x), I_{2}(x)$ and $I_{3}(x)$ when $x \in \Omega_{n}$.

Estimate of $I_{1}$. We first observe that, if $x \in \Omega_{n}$, then $x \notin \Omega\left(0, \theta^{-n}\right)$; thus, by assumption (G), we have $\Omega\left(x, \theta^{1-n}\right) \cap \Omega\left(0, \theta^{1-n}\right)=\varnothing$, whence

$$
\Gamma(x, y) \leq \theta^{n-1} \quad \text { for every } y \in \overline{\Omega\left(0, \theta^{1-n}\right)} .
$$

From this, we obtain the following estimate for $I_{1}(x)$ :

$$
\begin{aligned}
I_{1}(x) & \leq \theta^{n-1} \mu\left(\overline{\Omega\left(0, \theta^{1-n}\right)}\right) \leq \frac{1}{\theta} \cdot\left(\theta^{n} \mu\left(\Omega\left(0, \theta^{-n}\right)\right)\right) \\
& =\frac{1}{\theta} \cdot\left(\mu\left(\Omega\left(0, \theta^{-n}\right)\right) \int_{\theta^{-n}}^{\infty} \frac{1}{r^{2}} \mathrm{~d} r\right) \\
& \leq \frac{1}{\theta} \cdot \int_{\theta^{-n}}^{\infty} \frac{\mu(\Omega(0, r))}{r^{2}} \mathrm{~d} r .
\end{aligned}
$$


We explicitly point out that, as a consequence of Theorem 3.1 the integral in the far left-hand side of the above inequality is finite.

Estimate of $I_{3}$. Let $y \in \mathbb{R}^{N}$ be such that $\Gamma_{0}(y) \leq \theta^{n+2}$ and let

$$
\rho_{y}=\left(\Gamma_{0}(y)\right)^{-1}>0 \text {. }
$$

Since, obviously, $y \notin \Omega\left(0, \rho_{y}\right)$, assumption $(\mathrm{G})$ implies that the $\Gamma$-balls $\Omega\left(0, \theta \rho_{y}\right)$ and $\Omega\left(y, \theta \rho_{y}\right)$ are disjoint; on the other hand, if $x \in \Omega_{n}$, one has

$$
\Gamma_{0}(x)>\theta^{n+1}=\frac{1}{\theta} \cdot \theta^{n+2} \geq \frac{\Gamma_{0}(y)}{\theta}=\left(\theta \rho_{y}\right)^{-1},
$$

and thus $x \in \Omega\left(0, \theta \rho_{y}\right)$. As a consequence, we derive that $x \notin \Omega\left(y, \theta \rho_{y}\right)$, whence

$$
\Gamma(y, x)=\Gamma(x, y) \leq\left(\theta \rho_{y}\right)^{-1}=\frac{\Gamma(0, y)}{\theta} .
$$

By exploiting this last estimate, we obtain

$$
\begin{aligned}
I_{3}(x) & \leq \frac{1}{\theta} \cdot \int_{\left\{\Gamma_{0}(y) \leq \theta^{n+2}\right\}} \Gamma_{0}(y) \mathrm{d} \mu(y) \\
& =\frac{1}{\theta} \cdot \int_{\left\{\Gamma_{0}(y) \leq \theta^{n+2}\right\}}\left(\int_{1 / \Gamma_{0}(y)}^{\infty} \frac{1}{r^{2}} \mathrm{~d} r\right) \mathrm{d} \mu(y) \\
& \leq \frac{1}{\theta} \cdot \int_{\theta^{-n-2}}^{\infty} \frac{\mu(\Omega(0, r))}{r^{2}} \mathrm{~d} r \\
& \leq \frac{1}{\theta} \cdot \int_{\theta^{-n}}^{\infty} \frac{\mu(\Omega(0, r))}{r^{2}} \mathrm{~d} r .
\end{aligned}
$$

Estimate of $I_{2}$. The estimate of $I_{2}(x)$ (when $x \in \Omega_{n}$ ) is the crucial part of the proof. To begin with, we fix $p \in(1, \infty)$ and we define

$$
\begin{aligned}
& \mu_{n}:=\mu\left(\left\{y \in \mathbb{R}^{N}: \theta^{n+2}<\Gamma_{0}(y)<\theta^{n-1}\right\}\right) ; \\
& \eta_{n}:=\mu_{n} \theta^{n} \\
& \varepsilon_{n}:=\eta_{n}^{1-1 / p} .
\end{aligned}
$$

We claim that series $\sum_{n=1}^{\infty} \eta_{n}$ is convergent. In fact, for every $n \in \mathbb{N}$ we have

$$
\begin{aligned}
\eta_{n} \leq & \frac{1}{\theta^{2}} \cdot \int_{\left\{\theta^{n+2}<\Gamma_{0}(y)<\theta^{n-1}\right\}} \Gamma_{0}(y) \mathrm{d} \mu(y) \\
\leq & \frac{1}{\theta^{2}} \cdot\left(\int_{\left\{\Gamma_{0}(y) \leq \theta^{n-1}\right\}}-\int_{\left\{\Gamma_{0}(y) \leq \theta^{n+2}\right\}}\right) \Gamma_{0}(y) \mathrm{d} \mu(y) \\
\leq \frac{1}{\theta^{2}} \cdot\{ & \int_{\theta^{1-n}}^{\theta^{-2-n}} \frac{\mu(\Omega(0, r))}{r^{2}} \mathrm{~d} r \\
& \left.+\left(\theta^{n+2} \mu\left(\Omega\left(0, \theta^{-2-n}\right)\right)-\theta^{n-1} \mu\left(\Omega\left(0, \theta^{1-n}\right)\right)\right)\right\} .
\end{aligned}
$$


On the other hand, by arguing as for the estimate of $I_{1}(x)$, we see that

$$
r \mu(\Omega(0, r)) \leq \int_{r}^{\infty} \frac{\mu(\Omega(0, t))}{t^{2}} \mathrm{~d} t \longrightarrow 0 \quad \text { as } r \rightarrow \infty ;
$$

as a consequence, we obtain

$$
\begin{aligned}
& \sum_{n=1}^{\infty}\left(\theta^{n+2} \mu\left(\Omega\left(0, \theta^{-2-n}\right)\right)-\theta^{n-1} \mu\left(\Omega\left(0, \theta^{1-n}\right)\right)\right) \\
& \quad=-\left(\theta^{2} \mu\left(\Omega\left(0, \theta^{-2}\right)\right)+\theta \mu\left(\Omega\left(0, \theta^{-1}\right)\right)+\mu(\Omega(0,1))\right)<0 .
\end{aligned}
$$

Gathering together all these facts, we conclude that (see Theorem 3.1)

$$
\sum_{n=1}^{\infty} \eta_{n} \leq \frac{1}{\theta^{2}} \cdot \int_{1}^{\infty} \frac{\mu(\Omega(0, r))}{r^{2}} \mathrm{~d} r<\infty
$$

as claimed. In particular, we have $\eta_{n} \longrightarrow 0$ as $n \rightarrow \infty$.

We now observe that, if we consider the Radon measure $\lambda_{n}$ defined by

$$
\mathrm{d} \lambda_{n}=\mathbf{1}_{\left\{\theta^{n+2}<\Gamma_{0}<\theta^{n-1}\right\}} \mathrm{d} \mu,
$$

we have $\lambda_{n}\left(\mathbb{R}^{N}\right)=\mu_{n}<\infty$ and, for every $x \in \mathbb{R}^{N}$, we can write

$$
I_{2}(x)=\int_{\mathbb{R}^{N}} \Gamma(x, y) \mathrm{d} \lambda_{n}(y)=\Gamma \lambda_{n}(x) .
$$

By Lemma 3.5, it is then possible to find a family $\mathcal{F}_{n}=\left\{\overline{\Omega\left(x_{k, n}, r_{k, n}\right)}\right\}_{k \in J_{n}}$ (with $J_{n} \subseteq \mathbb{N}$ ) of closed $\Gamma$-balls satisfying the following properties:

(i) $\left\{x \in \Omega_{n}: I_{2}(x)<\varepsilon_{n}\right\} \supseteq \Omega_{n} \backslash \bigcup_{k \in J_{n}} \overline{\Omega\left(x_{k, n}, r_{k, n}\right)}$;

(ii) $\sum_{k \in J_{n}}\left(r_{k, n}\right)^{p}<A_{p}\left(\mu_{n} / \varepsilon_{n}\right)^{p}$ for a suitable constant $A_{p}>0$.

As a consequence of property (ii), for every $k \in J_{n}$ we have

$$
r_{k, n} \leq A_{p}^{1 / p}\left(\mu_{n} / \varepsilon_{n}\right) \stackrel{(3.9)}{=}\left(A_{p} \eta_{n}\right)^{1 / p} \theta^{-n}
$$

moreover, by property (i), we can assume that $\Omega\left(x_{k, n}, r_{k, n}\right) \cap \Omega_{n} \neq \varnothing$ for every index $k \in J_{n}$. This implies the existence of $n_{0} \in \mathbb{N}$ such that

$$
\Gamma_{0}\left(x_{k, n}\right) \leq \theta^{n-2} \quad \text { for every } n \geq n_{0} \text { and every } k \in J_{n} .
$$

Indeed, if $z$ is any point in $\Omega\left(x_{k, n}, r_{k, n}\right) \cap \Omega_{n} \subseteq \Omega_{n}$, we see that $z \notin \Omega\left(0, \theta^{-n}\right)$ and thus, again by assumption $(\mathrm{G})$, we infer that

$$
\Omega\left(z, \theta^{1-n}\right) \cap \Omega\left(0, \theta^{1-n}\right)=\varnothing .
$$

On the other hand, since $z$ also belongs to $\Omega\left(x_{k, n}, r_{k, n}\right)$, by (3.10) one has

$$
\Gamma\left(x_{k, n}, z\right)=\Gamma_{z}\left(x_{k, n}\right)>\frac{1}{r_{k, n}} \geq \theta^{n}\left(A_{p} \eta_{n}\right)^{-1 / p} ;
$$


as a consequence, if $n_{0} \in \mathbb{N}$ is such that $\left(A_{p} \eta_{n}\right)^{-1 / p}>\theta^{-1}$ for every $n \geq n_{0}$ (note that $\eta_{n} \rightarrow 0$ as $n \rightarrow \infty$ and $\left.-1 / p<0\right)$, we derive that $\Gamma_{z}\left(x_{k, n}\right)>\theta^{n-1}$, whence $x_{k, n} \in \Omega\left(z, \theta^{1-n}\right)$, and thus $x_{k, n} \notin \Omega\left(0, \theta^{1-n}\right)$. This implies that

$$
\Gamma_{0}\left(x_{k, n}\right) \leq \theta^{n-1}<\theta^{n-2} \quad \text { for every } n \geq n_{0} \text { and every } k \in J_{n} .
$$

By combining this last estimate with the choice of $\varepsilon_{n}$ and property (ii), we get

$$
\begin{gathered}
\sum_{n=n_{0}}^{\infty} \sum_{k \in J_{n}}\left(\Gamma_{0}\left(x_{k, n}\right) r_{k, n}\right)^{p} \leq\left(A_{p} \theta^{-2 p}\right) \sum_{n=n_{0}}^{\infty} \theta^{p n}\left(\frac{\mu_{n}}{\varepsilon_{n}}\right)^{p} \\
=\left(A_{p} \theta^{-2 p}\right) \cdot \sum_{n=n_{0}}^{\infty} \eta_{n}<\infty .
\end{gathered}
$$

Furthermore, by collecting the estimates for $I_{1}(x), I_{2}(x)$ and $I_{3}(x)$, we obtain

$$
u_{0}-u(x) \leq \frac{2}{\theta} \cdot \int_{\theta^{-n}}^{\infty} \frac{\mu(\Omega(0, r))}{r^{2}} \mathrm{~d} r+\eta_{n}^{1-1 / p}
$$

for every $x \in \Omega_{n}$ such that $I_{2}(x)<\varepsilon_{n}$. We finally claim that the set

$$
F:=\bigcup_{n \geq n_{0}} \bigcup_{k \in J_{n}} \Omega\left(x_{k, n}, r_{k, n}\right)
$$

is $p_{\mathcal{L}}$-bounded and it satisfies (1.5). In fact, if we introduce the family

$$
\mathcal{F}:=\left\{\Omega\left(x_{k, n}, r_{k, n}\right)\right\}_{\substack{n \geq n_{0} \\ k \in J_{n}}}
$$

we derive from (3.11) that $\mathcal{F}$ (which is obviously a countable cover of $F$ ) satisfies property (b) in Definition 1.4 hence $F$ is $p_{\mathcal{L}}$-bounded; moreover, since $\theta^{-n} \rightarrow \infty$ and $\eta_{n} \rightarrow 0$ as $n \rightarrow \infty$ (note that $\theta<1$ and $p>1$ ), for every $\varepsilon>0$ it is possible to find $n_{\varepsilon} \geq n_{0}$ such that (see also (3.4) in Theorem 3.1)

$$
\frac{2}{\theta} \cdot \int_{\theta^{-n}}^{\infty} \frac{\mu(\Omega(0, r))}{r^{2}} \mathrm{~d} r+\eta_{n}^{1-1 / p}<\varepsilon \quad \text { for every } n \geq n_{\varepsilon} .
$$

On the other hand, for every $x \in \mathbb{R}^{N} \backslash \overline{\Omega\left(0, \theta^{-n_{\varepsilon}}\right)}$ (which is an open neighborhood of $\infty$ ) non belonging to $F$, there exists a (unique) $n \geq n_{\varepsilon} \geq n_{0}$ such that

$$
x \in \Omega_{n} \backslash \bigcup_{k \in J_{n}} \Omega\left(x_{k, n}, r_{k, n}\right) \subseteq\left\{z \in \mathbb{R}^{N}: I_{2}(z)<\varepsilon_{n}\right\} ;
$$

as a consequence, by combining (3.12) with (3.13) we conclude that

$$
0 \leq u_{0}-u(x)<\varepsilon \text { for every } x \in \mathbb{R}^{N} \backslash F \text { with } \Gamma_{0}(x) \leq \theta^{-n_{\varepsilon}} .
$$

This shows that (1.5) holds true, and the proof is complete.

Now we have established Theorem 1.6, we can finally prove Theorem 1.5

Proof (of Theorem 1.5). We demonstrate the following equivalent fact: if $F \subseteq \mathbb{R}^{N}$ is $\mathcal{L}$-thin at infinity, then $F$ is $p_{\mathcal{L}}$-bounded for any $p>1$. 
To this end, we let $F \subseteq \mathbb{R}^{N}$ be $\mathcal{L}$-thin at infinity and, by contradiction, we

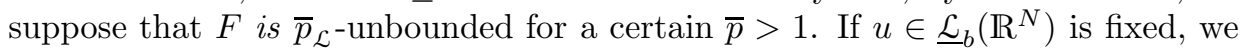

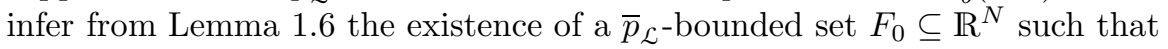

$$
\lim _{\substack{x \rightarrow \infty \\ x \notin F}} u(x)=\sup _{\mathbb{R}^{N}} u
$$

moreover, since $F_{0}$ is $\bar{p}_{\mathcal{L}}$-bounded but $F$ is not, then $F \backslash F_{0} \subseteq F$ is $\bar{p}_{\mathcal{L}}$-unbounded. In particular, $F \backslash F_{0}$ is non-void and unbounded (see (2) and (4) in Remark 3.4). By combining this last fact with (3.14), we then obtain

$$
\lim _{\substack{x \rightarrow \infty \\ x \in F \backslash F_{0}}} u(x)=\sup _{\mathbb{R}^{N}} u
$$

which obviously implies that

$$
\sup _{F} u \geq \limsup _{\substack{x \rightarrow \infty \\ x \in F}} u(x) \geq \lim _{\substack{x \rightarrow \infty \\ x \in F \backslash F_{0}}} u(x)=\sup _{\mathbb{R}^{N}} u .
$$

Owing to Corollary 2.3 , we conclude that $F$ is $\mathcal{L}$-large at infinity, which is in contradiction with our assumption on $F$. This ends the proof.

\section{$4 \quad \Gamma$-cones}

The present section is aimed to demonstrate the criterion for $p_{\mathcal{L}}$-unboundedness contained in Theorem 1.8. To this end, as anticipated in the Introduction, we need to require our PDOs $\mathcal{L}$ to satisfy another additional assumption:

(D) there exist two constants $\alpha^{\prime}, \alpha^{\prime \prime}>2$, with $\alpha^{\prime}<\alpha^{\prime \prime}$, such that

$$
\alpha^{\prime}|\Omega(x, r)| \leq|\Omega(x, 2 r)| \leq \alpha^{\prime \prime}|\Omega(x, r)|
$$

for every $x \in \mathbb{R}^{N}$ and every $r>0$ (here and throughout, $|A|$ indicate the standard $N$-dimensional Lebesgue measure in $\mathbb{R}^{N}$ of a Borel set $A \subseteq \mathbb{R}^{N}$ ).

Roughly put, assumption (D) represents a global doubling and reverse doubling condition for the $N$-volume of $\Gamma$-balls; as we shall see in the next Section [5] such a condition is fulfilled when homogeneous Hörmander operators are involved.

Remark 4.1. It is not difficult to recognize that (4.1) in assumption (D) implies the following crucial fact: there exists a constant $\alpha \geq 1$ such that

$$
\frac{1}{\alpha}\left(\frac{R}{r}\right)^{p}|\Omega(x, r)| \leq|\Omega(x, R)| \leq \alpha\left(\frac{R}{r}\right)^{q}|\Omega(x, r)|
$$

for every $x \in \mathbb{R}^{N}$ and every $0<r<R$, where

$$
p:=\log _{2}\left(1 / \alpha^{\prime}\right)>1 \quad \text { and } \quad q=\log _{2}\left(\alpha^{\prime \prime}\right)>1 .
$$

As will be clear from the sequel, the rôle of assumption (D) is only to guarantee the validity of (4.2) with $p>1$ : in fact, in what follows we shall only use this relation, which could also be taken as an assumption (in place of (4.1)).

Notice that, if (4.2) holds true (for some $\alpha \geq 1$ and $p, q>1$ ), by taking $R=2 r$ one re-obtains (4.1) with $\alpha^{\prime}=\alpha / 2^{p}$ and $\alpha^{\prime \prime}=2^{q} \alpha$; however, if we do not have any information on the value of $\alpha$, we cannot expect that $\alpha^{\prime}>2$. Thus, in some sense, the validity of (4.2) is a weaker assumption if compared to (D). 
With assumption (D) at hand, the proof of Theorem [1.8 will easily follow by combining Remark 3.4 with the next non-trivial result.

Theorem 4.2. Let $K \subseteq \mathbb{R}^{N}$ be a $\Gamma$-cone, according to Definition 1.7. Then $K$ is $p_{\mathcal{L}}$-unbounded (for the same $p>1$ in (4.3)).

Proof. Let $\mathcal{F}=\left\{\Omega\left(z_{j}, R_{j}\right\}_{j \in J}\right.$ be a family of $\Gamma$-balls contained in $K$ satisfying (i) and (ii) in Definition [1.7 Moreover, let c be the constant appearing in the pseudo-triangle inequality for $\gamma$ (see Remark 3.2) and let $M:=4 \mathbf{c}^{2}>4$.

Since $\gamma_{0}(z)=1 / \Gamma_{0}(z) \rightarrow \infty$ as $\|z\| \rightarrow \infty$ (see assumption (FS)-(d)), properties (i) and (ii) of $\mathcal{F}$ imply the existence of an increasing sequence $\left\{k_{j}\right\}_{j \in \mathbb{N}}$ of natural numbers and a real $\delta \in(0,1 / M)$ such that

(a) $\gamma_{0}\left(z_{k_{j+1}}\right)>M^{2} \gamma_{0}\left(z_{k_{j}}\right)$ for every $j \in \mathbb{N}$;

(b) $R_{k_{j}} \geq \delta \gamma_{0}\left(z_{k_{j}}\right)$ for every $j \in \mathbb{N}$.

We then set, for every natural $j$,

$$
y_{j}:=z_{k_{j}}, \quad \rho_{j}:=\delta \gamma_{0}\left(z_{k_{j}}\right), \quad B_{j}:=\Omega\left(y_{j}, \rho_{j}\right)
$$

and we consider the set $F_{0}$ defined as follows:

$$
F_{0}:=\bigcup_{j=1}^{\infty} B_{j} .
$$

Since, by (b), $R_{k_{j}} \geq \rho_{j}$, we derive that $B_{j} \subseteq \Omega\left(z_{k_{j}}, R_{k_{j}}\right) \subseteq K$ for every $j \in \mathbb{N}$; hence, $F_{0} \subseteq K$. As a consequence, to prove that $K$ is $p_{\mathcal{L}}$-unbounded it suffices to show that $F_{0}$ is $p_{\mathcal{L}}$-unbounded (for the same $p>1$ appearing in (4.3)).

To this end, we choose a sequence $\left\{D_{n}=\Omega\left(x_{n}, r_{n}\right)\right\}_{n}$ of $\Gamma$-balls such that

$$
F_{0} \subseteq \bigcup_{n=1}^{\infty} D_{n}
$$

and we prove that, if $p$ is as in (4.3), one has (see Definition 1.4)

$$
\sum_{n=1}^{\infty}\left(\Gamma_{0}\left(x_{n}\right) r_{n}\right)^{p}=\sum_{n=1}^{\infty}\left(\frac{r_{n}}{\gamma_{0}\left(x_{n}\right)}\right)^{p}=\infty .
$$

Let then $\varepsilon \in(0,1 / M)$ be fixed and let $\mathcal{A}_{\varepsilon} \subseteq \mathbb{N}$ be defined as follows:

$$
\mathcal{A}_{\varepsilon}:=\left\{n \in \mathbb{N}: \frac{r_{n}}{\gamma_{0}\left(x_{n}\right)} \geq \varepsilon\right\} .
$$

If $\mathcal{A}_{\varepsilon}$ is infinite, then the claimed (4.5) is obviously true; we thus assume that the set $\mathcal{A}_{\varepsilon}$ is finite and we choose a natural $\bar{n}=\bar{n}_{\varepsilon}$ such that

$$
\frac{r_{n}}{\gamma_{0}\left(x_{n}\right)} \leq \varepsilon \quad \text { for every } n \geq \bar{n} \text {. }
$$

We now prove some technical facts we shall need to show that (4.5) holds.

Claim I: There exists a natural $\bar{j}=\bar{j}_{\varepsilon}$ such that

$$
\bigcup_{j \geq \bar{j}} B_{j} \subseteq \bigcup_{n \geq \bar{n}} D_{n} .
$$


In fact, let $k \in \mathbb{N}$ be such that $B_{k} \cap D_{n} \neq \varnothing$ for some $n \in \mathcal{J}:=\{1, \ldots, \bar{n}-1\}$ and let $z \in B_{k} \cap D_{n}$, By the properties of $\gamma$ in Remark 3.3 we get

$$
\begin{aligned}
\rho_{k} & \stackrel{\text { 4.4) }}{=} \delta \gamma_{0}\left(y_{k}\right) \leq \delta \mathbf{c}\left(\gamma(0, z)+\gamma\left(z, y_{k}\right)\right) \\
& \leq \delta \mathbf{c}^{2}\left(\gamma\left(0, x_{n}\right)+\gamma\left(x_{n}, z\right)+\gamma\left(z, y_{k}\right)\right) \\
& \leq \delta \mathbf{c}^{2}\left(\rho_{k}+\max _{n \in \mathcal{J}}\left(r_{n}+\gamma_{0}\left(x_{n}\right)\right)\right) \quad\left(\text { since } z \in B_{k} \cap D_{n}\right)
\end{aligned}
$$

as a consequence, since $\delta \mathbf{c}^{2}<1 / 4<1$ ( by the choice of $\delta$ ), we obtain

$$
\rho_{k} \leq \frac{\max _{n \in \mathcal{J}}\left(r_{n}+\gamma_{0}\left(x_{n}\right)\right)}{1-\delta \mathbf{c}}=: \tau \text {. }
$$

On the other hand, since $\rho_{j}=\delta \gamma_{0}\left(y_{j}\right) \rightarrow \infty$ as $j \rightarrow \infty$ (by (b)), it is possible to find a natural $\bar{j}=\bar{j}_{\varepsilon}$ such that $\rho_{j}>\tau$ for every $j \geq \bar{j}$; hence

$$
B_{j} \cap D_{n}=\varnothing \quad \text { for every } j \geq \bar{j} \text { and every } n<\bar{n} \text {. }
$$

By taking into account that $\left\{D_{n}\right\}_{n}$ is a cover of $F_{0}$, we obtain (4.7).

Claim II: If $\bar{j} \in \mathbb{N}$ is as in (4.7), we define

$$
P_{j}:=\left\{n \geq \bar{n}: B_{j} \cap D_{n} \neq \varnothing\right\} .
$$

Then the following fats hold true:

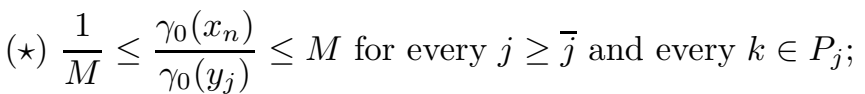

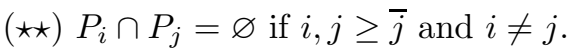

As for $(\star)$ we observe that, if $n \in P_{j}$ (for some $j \geq \bar{j}$ ) and if $z \in B_{j} \cap D_{n} \neq \varnothing$, by the properties of $\gamma$ in Remark 3.3 (and the choice of $M$ and $\varepsilon$ ) we have

$$
\begin{aligned}
\gamma_{0}\left(x_{n}\right) & =\gamma\left(0, x_{n}\right) \leq \mathbf{c}\left(\gamma\left(x_{n}, z\right)+\gamma(0, z)\right) \\
& \leq \mathbf{c}\left(\gamma\left(x_{n}, z\right)+\mathbf{c}\left(\gamma\left(0, y_{j}\right)+\gamma\left(y_{j}, z\right)\right)\right) \\
& \leq \mathbf{c}^{2}\left(r_{n}+\rho_{j}+\gamma_{0}\left(y_{j}\right)\right) \quad\left(\text { since } z \in B_{j} \cap D_{n}\right) \\
& \left.\leq \mathbf{c}^{2}\left((1+\delta) \gamma_{0}\left(y_{j}\right)+\varepsilon \gamma_{0}\left(x_{n}\right)\right) \quad \text { (see (14.4) and (4.6) }\right) \\
& \leq 2 \mathbf{c}^{2} \gamma_{0}\left(y_{j}\right)+\frac{1}{2} \gamma_{0}\left(x_{n}\right) .
\end{aligned}
$$

From this, we derive that

$$
\frac{\gamma_{0}\left(x_{n}\right)}{\gamma_{0}\left(y_{j}\right)} \leq 4 \mathbf{c}^{2}=M
$$

which is precisely the second inequality in $(\star)$; by arguing analogously, one can prove the first one too. We now turn to prove $(\star \star)$.

To this end, we argue by contradiction and we assume the existence of two indexes $i, j \geq \bar{j}$, with (to fix ideas) $j>i$, such that $P_{i} \cap P_{j} \neq \varnothing$. If $n \geq \bar{n}$ is a natural number belonging to such an intersection, from $(\star)$ we derive that

$$
\frac{\gamma_{0}\left(y_{j}\right)}{\gamma_{0}\left(y_{i}\right)}=\frac{\gamma_{0}\left(y_{j}\right)}{\gamma_{0}\left(x_{n}\right)} \cdot \frac{\gamma_{0}\left(x_{n}\right)}{\gamma_{0}\left(y_{i}\right)} \leq M^{2} \text {, }
$$


which is in contradiction with (a).

Claim III: There exists a real constant $\zeta>0$ such that

$$
\left(\frac{\gamma_{0}\left(y_{j}\right)}{r_{n}}\right)^{p} \cdot \frac{\left|D_{n}\right|}{\left|B_{j}\right|} \leq \zeta \quad \text { for any } j \geq \bar{j} \text { and } n \in P_{j} .
$$

In fact, by using $(\star)$ and the pseudo-triangle inequality for $\gamma$, it is possible to find a real $\zeta_{1}>1$, only depending on $M$ and $\mathbf{c}$, such that

$$
\Omega\left(x_{n}, \gamma_{0}\left(y_{j}\right)\right) \subseteq \Omega\left(y_{j}, \zeta_{1} \gamma_{0}\left(y_{j}\right)\right) \text { for any } j \geq \bar{j} \text { and } n \in P_{j} .
$$

From this, by applying the second inequality in (4.2), we obtain

$$
\begin{aligned}
\frac{\left|D_{n}\right|}{\left|B_{j}\right|} & =\frac{\left|\Omega\left(x_{n}, r_{n}\right)\right|}{\left|\Omega\left(y_{j}, \rho_{j}\right)\right|} \stackrel{(4.4)}{=} \frac{\left|\Omega\left(x_{n}, r_{n}\right)\right|}{\mid \Omega\left(y_{j},\left(\delta / \zeta_{1}\right) \cdot\left(\zeta_{1} \gamma_{0}\left(y_{j}\right)\right) \mid\right.} \\
& \leq \alpha\left(\frac{\zeta_{1}}{\delta}\right)^{q} \cdot \frac{\left|\Omega\left(x_{n}, r_{n}\right)\right|}{\left|\Omega\left(y_{j}, \zeta_{1} \gamma_{0}\left(y_{j}\right)\right)\right|} \quad\left(\text { since } \delta / \zeta_{1}<1\right) \\
& \leq \alpha\left(\frac{\zeta_{1}}{\delta}\right)^{q} \cdot \frac{\left|\Omega\left(x_{n}, r_{n}\right)\right|}{\left|\Omega\left(x_{n}, \gamma_{0}\left(y_{j}\right)\right)\right|}:=(\star) \quad \text { (by (4.9)) }
\end{aligned}
$$

On the other hand, since $n \geq \bar{n}$, again by $(\star)$ we have

$$
\frac{r_{n}}{\gamma_{0}\left(y_{j}\right)} \leq \varepsilon \cdot \frac{\gamma_{0}\left(x_{n}\right)}{\gamma_{0}\left(y_{j}\right)} \leq \varepsilon M<1
$$

we are then entitled to use the reverse doubling condition (4.2), which gives

$$
(\star) \leq \zeta\left(\frac{r_{n}}{\gamma_{0}\left(y_{j}\right)}\right)^{p}
$$

for some universal constant $\zeta$ not depending on $j$ and $n$.

Now we have established all these claims, we can easily achieve the proof of the needed (4.5). Indeed, by $(\star)$ and $(\star \star)$ in Claim II, we have

$$
\sum_{n \geq \bar{n}}\left(\frac{r_{n}}{\gamma_{0}\left(x_{n}\right)}\right)^{p} \geq \sum_{j \geq \bar{j}} \sum_{n \in P_{j}}\left(\frac{r_{n}}{\gamma_{0}\left(x_{n}\right)}\right)^{p} \geq \frac{1}{M^{p}} \sum_{j \geq \bar{j}} \sum_{n \in P_{j}}\left(\frac{r_{n}}{\gamma_{0}\left(y_{j}\right)}\right)^{p}=:(\star)
$$

On the other hand, if $j \geq \bar{j}$ is fixed, the family $\left\{D_{n}\right\}_{n \in P_{j}}$ is a cover of the set $B_{j}$ (see (4.7)); as a consequence, by exploiting estimate (4.8), we finally obtain

$$
\begin{aligned}
(\star) & \geq\left(\zeta M^{p}\right)^{-1} \sum_{j \geq \bar{j}} \frac{1}{\left|B_{j}\right|} \sum_{n \in P_{j}}\left|D_{n}\right| \\
& \geq\left(\zeta M^{p}\right)^{-1} \sum_{j \geq \bar{j}} 1=\infty .
\end{aligned}
$$

This is precisely the desired (4.5), and the proof is complete.

With Theorem 4.2 at hand, we can now prove Theorem 1.8 .

Proof (of Theorem [1.8). Let $F, K$ be as in the statement of the theorem. Since, by assumption, $K$ is a $\Gamma$-cone, we infer from Theorem 4.2 that $K$ is $p_{\mathcal{L}}$-unbounded (for the same $p$ as in (4.3)); on the other hand, as $K \subseteq F$, Remark 3.4.(2.) shows that also $F$ is $p_{\mathcal{L}}$-unbounded, and the proof is complete. 


\section{The case of homogeneous Hörmander operators}

The aim of this final section is to show that any Hörmander's operator sum of squares of homogeneous vector fields satisfies all the assumptions (H1)-to-(H3), (FS), (G), (L) and (D) introduced in the previous sections.

To this end, we fix once and for all a family $X=\left\{X_{1}, \ldots, X_{m}\right\}$ of linearly independent smooth vector fields on $\mathbb{R}^{N}$, with $N \geq 3$, satisfying assumptions (I) and (II) in the Introduction. Moreover, we let

$$
Q:=\sum_{j=1}^{N} \sigma_{j} \geq 3,
$$

be the homogeneous dimension of $\mathbb{R}^{N}$ with respect to the family of dilations

$$
\delta_{\lambda}: \mathbb{R}^{N} \rightarrow \mathbb{R}^{N}, \quad \delta_{\lambda}(x)=\left(\lambda^{\sigma_{1}} x_{1}, \ldots, \lambda^{\sigma_{N}} x_{N}\right) .
$$

We then denote by $\mathcal{L}$ the operator naturally associated with $\chi$, that is,

$$
\mathcal{L}=\sum_{j=1}^{m} X_{j}^{2} .
$$

Assumptions (H1)-to-(H3). It is easy to recognize that $\mathcal{L}$ satisfies all the structural assumptions (H1)-to-(H3) introduced in Section 1 indeed, a direct computation shows that $\mathcal{L}$ takes the form (1.1), with $V \equiv 1$ and

$$
A(x)=S(x) \cdot S(x)^{T}, \quad \text { where } S(x)=\left(X_{1}(x) \cdots X_{m}(x)\right) ;
$$

as a consequence, $\mathcal{L}$ is degenerate-elliptic. Moreover, the validity of Hörmander's Rank Condition easily implies that $\mathcal{L}$ is non-totally degenerate and that $\mathcal{L}$ is $C^{\infty}$. hypoelliptic on every open subset of $\mathbb{R}^{N}$ (by Hörmander's Theorem).

Assumption (FS). We now prove that $\mathcal{L}$ also satisfies assumption (FS). First of all, by exploiting [8, Theorem 1.1], we get the existence of a function $\Gamma(x ; y)$, defined out of the diagonal of $\mathbb{R}^{N} \times \mathbb{R}^{N}$, such that

- $\Gamma$ is smooth and strictly positive on its domain of definition;

- $\Gamma(x ; y)=\Gamma(y ; x)$ for every $x, y \in \mathbb{R}^{N}$ with $x \neq y$;

- for every fixed $x \in \mathbb{R}^{N}, y \mapsto \Gamma(x ; y)=\Gamma_{x}(y) \in L_{\text {loc }}^{1}\left(\mathbb{R}^{N}\right)$ and

$$
\int_{\mathbb{R}^{N}} \Gamma(x ; y) \mathcal{L} \varphi(y) \mathrm{d} y=-\varphi(x) \quad \text { for every } \varphi \in C_{0}^{\infty}\left(\mathbb{R}^{N}, \mathbb{R}\right) ;
$$

- $\Gamma(x ; \cdot)$ vanishes at infinity (uniformly for $x$ in compact sets);

- $\Gamma$ has the (joint) homogeneity property

$$
\Gamma\left(\delta_{\lambda}(x) ; \delta_{\lambda}(y)\right)=\lambda^{2-Q} \Gamma(x ; y) \text { for all } x, y \in \mathbb{R}^{N} .
$$

Furthermore, by using the results in the very recent paper [9] (see, precisely, Theorem $1.3-(\mathrm{V}))$, we know that $\Gamma(x ; \cdot)$ has a pole at $x$, i.e.,

$$
\lim _{y \rightarrow x} \Gamma(x ; y)=\infty \quad \text { for any fixed } x \in \mathbb{R}^{N} .
$$

Summing up, $\mathcal{L}$ satisfies assumption (FS). 
Assumption (G). In this paragraph prove that $\mathcal{L}$ also satisfies assumption $(\mathrm{G})$. To this end, we first need to remind some results concerning the so-called control distance associated with the family $\mathcal{X}=\left\{X_{1}, \ldots, X_{m}\right\}$.

Let $f:[0, T] \rightarrow \mathbb{R}^{N}$ be a Lipschitz curve. We say that $f$ is $\mathcal{X}$-subunit if

$$
\langle\dot{f}(t), \xi\rangle \leq \sum_{j=1}^{m}\left\langle X_{j}(f(t)), \xi\right\rangle^{2}, \quad \text { for a.e. } t \in[0, T] \text { and every } \xi \in \mathbb{R}^{N} \text {. }
$$

Denoting by $\mathcal{S}(X)$ the set of all $X$-subunit curves, we can define

$$
d x(x, y):=\inf \{T>0: \exists f \in \mathcal{S}(X) \text { such that } f(0)=x \text { and } f(T)=y\} .
$$

Since $X_{1}, \ldots, X_{m}$ satisfy Hörmander's Rank Condition, the function $d x$ is finite for every $x, y \in \mathbb{R}^{N}$ and it defines a distance on $\mathbb{R}^{N}$, which is usually referred to as the control distance associated with $X$ (see, e.g., [13, Chapter 19] and the references therein). Moreover, since the $X_{j}$ s are $\delta_{\lambda}$-homogeneous of degree 1 ,

$$
d_{x}\left(\delta_{\lambda}(x), \delta_{\lambda}(y)\right)=\lambda d_{x}(x, y) .
$$

For every fixed $x \in \mathbb{R}^{N}$ and every $r>0$, we indicate by $B x(x, r)$ the (open) $d x$-ball with centre $x$ and radius $r$, that is,

$$
B_{x}(x, r):=\left\{y \in \mathbb{R}^{N}: d_{x}(x, y)<r\right\} .
$$

By (5.2), it is easy to see that $d x$-balls are preserved by dilations, that is,

$$
\delta_{\lambda}\left(B_{X}(x, r)\right)=B_{X}\left(\delta_{\lambda}(x), \lambda r\right) ;
$$

from this, by using a deep result by Nagel, Stein and Wainger [20], one obtains the following global estimates for the $N$-volume of $d x$-balls (see also [9, Thm. B]).

Theorem 5.1. There exist a real constant $c_{1} \geq 1$ such that

$$
\frac{1}{c_{1}} \sum_{j=N}^{Q} F_{j}(x) r^{j} \leq\left|B_{x}(x, r)\right| \leq c_{1} \sum_{j=N}^{Q} F_{j}(x) r^{j}
$$

for every $x \in \mathbb{R}^{N}$ and every $r>0$. Here, the functions $F_{j}$ are positive continuous functions and, for every $j, F_{j}$ is $\delta_{\lambda}$-homogeneous of degree $Q-j$.

Proof. First of all, we need to introduce some notations borrowed from [20] (see also [14, Section 4.2]): if $p \in \mathbb{N}$ and $I=\left(i_{1}, \ldots, i_{p}\right)$ is a multi-index of length $p$ (i.e., $I$ is a vector in $\mathbb{R}^{p}$ with non-negative integer components), we define

$$
X_{I}:=\left[X_{i_{1}} \cdots\left[X_{i_{p-1}}, X_{i_{p}}\right] \cdots\right] \text { and }|I|:=p .
$$

Furthermore, if $B=\left(I_{1}, \ldots, I_{N}\right)$ is a $N$-tuple of multi-indexes, we set

$$
\lambda_{B}(x):=\operatorname{det}\left(X_{I_{1}}(x) \cdots X_{I_{N}}(x)\right) \quad \text { and } \quad l(B):=\sum_{j=1}^{N}\left|I_{j}\right| \geq N .
$$

Finally, if $s$ is any natural number, we denote by $\mathcal{B}_{s}$ the set of all the possible $N$-tuples $B=\left(I_{1}, \ldots, I_{N}\right)$ of multi-indexes with $\left|I_{j}\right| \leq s$ for every $j=1, \ldots, N$. 
We now observe that, by assumptions (H1) and (H2), the Lie algebra generated by $X_{1}, \ldots, X_{m}$ is nilpotent of step $s:=\sigma_{N}$; as a consequence, if $\mathcal{U} \subseteq \mathbb{R}^{N}$ is any (fixed) bounded and connected open neighborhood of 0, 20, Theorem 1] provides us with a small $r_{0}>0$ and a real constant $c_{1} \geq 1$ such that

$$
\frac{1}{c_{1}} \sum_{B \in \mathcal{B}_{s}}\left|\lambda_{B}(x)\right| r^{l(B)} \leq\left|B_{x}(x, r)\right| \leq c_{1} \sum_{B \in \mathcal{B}_{s}}\left|\lambda_{B}(x)\right| r^{l(B)}
$$

for every $x \in \mathcal{U}$ and every $r>0$ such that $r \leq r_{0}$.

We claim that, as a consequence of the homogeneity of $X_{1}, \ldots, X_{m}$, estimate (5.5) actually holds for every $x \in \mathbb{R}^{N}$ and every $r>0$. Indeed, if $x \in \mathbb{R}^{N}$ is arbitrary fixed and if $r>0$, it is possible to choose $\lambda=\lambda_{x, r}>0$ such that

$$
x^{\prime}=\delta_{\lambda}(x) \in \mathcal{U} \text { and } r^{\prime}=\lambda r \leq r_{0} ;
$$

thus, (5.5) holds with $x^{\prime}$ and $r^{\prime}$ in place of $x$ and $r$. Now, by (5.3) we have

$$
\left|B_{x}\left(x^{\prime}, \lambda r\right)\right|=\left|\delta_{\lambda}\left(B_{x}(x, r)\right)\right|=\lambda^{Q}\left|B_{x}(x, r)\right| ;
$$

on the other hand, if $B=\left(I_{1}, \ldots, I_{N}\right) \in \mathcal{B}_{s}$, the homogeneity of $X_{1}, \ldots, X_{m}$ with respect to $\delta_{\lambda}$ implies that (see, e.g., [13, Corollary 1.3.6])

$$
\begin{aligned}
& \left|\lambda_{B}\left(x^{\prime}\right)\right|\left(r^{\prime}\right)^{l(B)}=\left|\operatorname{det}\left(X_{I_{1}}\left(x^{\prime}\right) \cdots X_{I_{N}}\left(x^{\prime}\right)\right)\right|\left(r^{\prime}\right)^{l(B)} \\
& \quad=\operatorname{det}\left(\lambda^{-\left|I_{1}\right|} \delta_{\lambda}\left(X_{I_{1}}(x)\right) \cdots \lambda^{-\left|I_{N}\right|} \delta_{\lambda}\left(X_{I_{N}}(x)\right)\right)(\lambda r)^{l(B)} \\
& \quad=\lambda^{Q-l(B)} \lambda_{B}(x)(\lambda r)^{l(B)}=\lambda^{Q} \lambda_{B}(x) r .
\end{aligned}
$$

By combining (5.6) with (5.7), we conclude that the validity of (5.5) for $x^{\prime}$ and $r^{\prime}$ implies the validity of the same estimate for $x$ and $r$, as claimed.

To complete the demonstration of the theorem we observe that, if $B \in \mathcal{B}_{s}$, the computation carried out in (5.7) shows that $\left|\lambda_{B}\right|$ is a continuous $\delta_{\lambda}$-homogeneous function of degree $Q-l(B)$; as a consequence, we have

$$
\left|\lambda_{B}\right| \equiv 0 \quad \text { for every } B \in \mathcal{B}_{s} \text { with } l(B)>Q .
$$

Thanks to this last fact, we can write (for $x \in \mathbb{R}^{N}$ and $r>0$ )

$$
\sum_{B \in \mathcal{B}_{s}}\left|\lambda_{B}(x)\right| r^{l}(B)=\sum_{j=N}^{Q} \underbrace{\left(\sum_{\substack{B \in \mathcal{B}_{s} \\ l(B)=j}}\left|\lambda_{B}(x)\right|\right)}_{=: F_{j}(x)} r^{j} \equiv \sum_{j=N}^{Q} F_{j}(x) r^{j}
$$

Note that, by definition, any $F_{j}$ is $\delta_{\lambda}$-homogeneous of degree $Q-j$ (since $\left|\lambda_{B}\right|$ is $\delta_{\lambda}$-homogeneous of degree $Q-l(B)=Q-j$ if $\left.l(B)=j\right)$. This ends the proof.

We now turn to show how $d x$ (and the associated balls) are related with the fundamental solution $\Gamma$. To this end, we introduce the following functions:

$$
\Lambda(x, r):=\sum_{j=N}^{Q} F_{j}(x) r^{j}, \quad E(x, r):=\frac{\Lambda(x, r)}{r^{2}}=\sum_{j=N}^{Q} F_{j}(x) r^{j-2} .
$$


Remark 5.2. We list, for future reference, some useful properties of $\Lambda$ and $E$ :

(a) For every fixed $x$, both $\Lambda(x, \cdot)$ and $E(x, \cdot)$ are strictly increasing on $(0, \infty)$;

(b) For every $x \in \mathbb{R}^{N}$ and every $0<r<R$ we have

$$
\begin{aligned}
& \text { (b) } \quad\left(\frac{R}{r}\right)^{N} \Lambda(x, r) \leq \Lambda(x, R) \leq\left(\frac{R}{r}\right)^{Q} \Lambda(x, r) \\
& (\text { b })_{2} \quad\left(\frac{R}{r}\right)^{N-2} E(x, r) \leq E(x, R) \leq\left(\frac{R}{r}\right)^{Q-2} E(x, r)
\end{aligned}
$$

(c) If $B_{x}(x, r) \subseteq B_{x}(y, \rho)$ for some $x, y \in \mathbb{R}^{N}$ and $r, \rho \in(0, \infty)$, we have

$$
\Lambda(x, r) \leq c_{1}^{2} \Lambda(y, \rho) \quad \text { and } \quad E(x, r) \leq c_{1}^{2}\left(\frac{\rho}{r}\right)^{2} E(y, \rho) .
$$

(d) There exists a strictly positive constant $\omega_{Q}>0$ such that

$$
\begin{array}{lll}
(\mathrm{d})_{1} & \Lambda(0, r)=\omega_{Q} r^{Q} & \text { for every } r>0 ; \\
(\mathrm{d})_{2} & \Lambda(x, r) \geq \omega_{Q} r^{Q} & \text { for every } x \in \mathbb{R}^{N} \text { and every } r>0 .
\end{array}
$$

In fact, since any function $F_{j}$ appearing in (5.8) is non-negative, continuous and $\delta_{\lambda}$-homogeneous of degree $Q-j$ (see Theorem [5.1), we have

(*) $F_{j}(0)=0$ for every $j=N, \ldots, Q-1$;

$(* *) F_{Q}(x)=F_{Q}(0)=\omega_{Q} \geq 0$ for every $x \in \mathbb{R}^{N}$.

As a consequence, by the very definition of $\Lambda$ (see (5.8)), for every $x \in \mathbb{R}^{N}$ and for every $r>0$ we can write

$$
\Lambda(0, r)=\omega_{Q} r^{Q} \quad \text { and } \quad \Lambda(x, r) \geq \omega_{Q} r^{Q},
$$

with $\omega_{Q} \geq 0$. From this, since (5.4) implies that

$$
\omega_{Q}=\Lambda(0,1) \geq \frac{1}{c_{1}}\left|B_{x}(0,1)\right|>0,
$$

we obtain both (5.12) and (5.13).

By combining [9, Theorem 1.3-(III)] with the above Theorem [5.1] we are able to demonstrate the following key result.

Theorem 5.3. There exists a real constant $c_{2}>0$ such that

$$
\frac{1}{c_{2}} \frac{d_{x}^{2}(x, y)}{\Lambda\left(x, d_{x}(x, y)\right)} \leq \Gamma(x ; y) \leq c_{2} \frac{d_{x}^{2}(x, y)}{\Lambda\left(x, d_{x}(x, y)\right)} \quad \text { for all } x \neq y .
$$

Proof. First of all, since we are assuming that the operator $\mathcal{L}$ is defined on some space $\mathbb{R}^{N}$ with $N \geq 3$, we are entitled to apply [9, Theorem 1.3-(III)]: as a consequence, for every $x, y \in \mathbb{R}^{N}$ with $x \neq y$ we have

$$
C^{-1} \frac{d_{x}(x, y)^{2}}{\left|B_{x}\left(x, d_{x}(x, y)\right)\right|} \leq \Gamma(x ; y) \leq C \frac{d_{x}(x, y)^{2}}{\left|B_{x}\left(x, d_{X}(x, y)\right)\right|},
$$

where $C \geq 1$ is a suitable structural constant. By combining (5.15) with the global estimate (5.4) for $\left|B_{x}(x, r)\right|$ (holding true for any $r>0$ ), we immediately obtain the desired (5.14) (with $c_{2}:=C \cdot c_{1}$ ). This ends the proof. 
With Theorem 5.3 at hand, we can now prove that $\mathcal{L}$ fulfills assumption $(\mathrm{G})$.

Proposition 5.4. L satisfies assumption $(\mathrm{G})$ introduced in Section 3 .

Proof. According to Remark 3.2. $\mathcal{L}$ fulfill assumption $(\mathrm{G})$ if and only if the reciprocal function $\gamma(x, y)=1 / \Gamma(x ; y)$ (with the convention $\gamma(x, x)=0$ ) satisfies a pseudo-triangle inequality; on the other hand, by Theorem 5.3 we have

$$
\frac{1}{c_{1}} E\left(x, d_{x}(x, y)\right) \leq \gamma(x, y) \leq c_{2} E(x, d x(x, y)) \quad \text { for every } x, y \in \mathbb{R}^{N} .
$$

Thus, to prove the proposition, it suffices to show that there exists $\mathbf{c}>1$ such that, for every $x, y, z \in \mathbb{R}^{N}$, the following inequality holds true:

$$
E\left(x, d_{x}(x, y)\right) \leq \mathbf{c}\left(E\left(x, d_{x}(x, z)\right)+E\left(z, d_{x}(z, y)\right)\right) .
$$

First of all we observe that, since $d x$ satisfies a genuine triangle inequality, for every $x, y, z \in \mathbb{R}^{N}$ we have (see Remark 5.2 (a))

$$
E\left(x, d_{x}(x, y)\right) \leq E\left(x, d_{x}(x, z)+d_{x}(z, y)\right)=:(\star)
$$

as a consequence, if $d_{x}(z, y) \leq d x(x, z)$, we obtain (see also Remark 5.2.(b))

$$
\begin{aligned}
(\star) & \leq E\left(x, 2 d_{x}(x, z)\right) \stackrel{5.100}{\leq} 2^{Q-2} E\left(x, d_{x}(x, z)\right) \\
& \leq 2^{Q-2}\left(E\left(x, d_{x}(x, z)\right)+E\left(z, d_{x}(z, y)\right)\right) .
\end{aligned}
$$

If, instead, $d_{x}(z, y)>d_{x}(x, z)$, from the obvious fact that $B_{x}\left(x, 2 d_{x}(z, y)\right)$ is included in $B x\left(z, 3 d_{x}(z, y)\right)$ we get (see also Remark 5.2 . (c))

$$
\begin{aligned}
(\star) & \leq E\left(x, 2 d_{x}(z, y)\right) \stackrel{\sqrt[5.11]{\leq}}{\leq} c_{1}^{2}\left(\frac{3}{2}\right)^{2} E\left(z, 3 d_{x}(z, y)\right) \\
& \stackrel{5.10}{\leq} c_{1}^{2}\left(\frac{3}{2}\right)^{2} 3^{Q-2} E\left(z, d_{x}(z, y)\right) \\
& \leq\left(\frac{3^{Q} c_{1}^{2}}{4}\right)\left(E\left(x, d_{x}(x, z)\right)+E\left(z, d_{x}(z, y)\right)\right) .
\end{aligned}
$$

Setting $\mathbf{c}:=\max \left\{2^{Q-2}, 3^{Q} c_{1}^{2} / 4\right\}$, we obtain the desired (5.16).

Assumption (L). In this paragraph we prove that $\mathcal{L}$ satisfies the Liouville-type theorem in assumption $(\mathrm{L})$ : a bounded $\mathcal{L}$-harmonic function on $\mathbb{R}^{N}$ is constant.

Proposition 5.5. Let $u \in \mathcal{L}\left(\mathbb{R}^{N}\right)$ be a L-harmonic function on $\mathbb{R}^{N}$. If $u$ is bounded (above or below), then $u$ is constant throughout $\mathbb{R}^{N}$.

One demonstration of Proposition 5.5 can be found in [18; however, we present below another prove of this result, which is almost self-contained.

Proof. By [8, Theorem 3.2], it is possible to find a homogeneous Carnot group $\mathbb{G}=\left(\mathbb{R}^{H}, *, d_{\lambda}\right)$ on $\mathbb{R}^{H}$ (for a suitable $H>N$ ) and a system $\mathcal{Z}=\left\{Z_{1}, \ldots, Z_{m}\right\}$ of Lie-generator for $\operatorname{Lie}(\mathbb{G})$ such that, setting $\Delta_{\mathbb{G}}=\sum_{j=1}^{m} Z_{j}^{2}$, one has

$$
\Delta_{\mathbb{G}}(f \circ \pi)=(\mathcal{L} f) \circ \pi \quad \text { for every } f \in C^{\infty}\left(\mathbb{R}^{N}, \mathbb{R}\right)
$$


(here, $\pi: \mathbb{R}^{H} \rightarrow \mathbb{R}^{N}$ is the canonical projection of $\mathbb{R}^{H}$ onto the first $N$ variables). Thus, since $u \in \mathcal{H}_{\mathcal{L}}\left(\mathbb{R}^{N}\right)$, the function $v:=u \circ \pi$ is $\Delta_{\mathbb{G}}$-harmonic on $\mathbb{G} \equiv \mathbb{R}^{H}$.

On the other hand, since (by assumption) $u$ is bounded (from above or from below), then the same is true of $v$; as a consequence, by the classical Liouville Theorem on Carnot groups (see, e.g., [13, Theorem 5.8.2]), we conclude that $v$ is constant throughout $\mathbb{R}^{H}$, whence $u$ is constant on $\mathbb{R}^{N}$. This ends the proof.

Assumption (D). In this last paragraph of the section we prove that $\mathcal{L}$ fulfills assumption (D). Actually, according to Remark 4.1, we directly show that the super-level sets of $\Gamma$ satisfy the doubling/reverse doubling conditions in (4.2).

To this end we first observe that, since the function $E(x, \cdot)$ is strictly increasing on $(0, \infty)$ for every fixed $x \in \mathbb{R}^{N}$ (see Remark [5.2), we can define

$$
H(x, \cdot):=(E(x, \cdot))^{-1} \quad \text { on }(0, \infty) .
$$

Obviously, $H$ is strictly increasing on $(0, \infty)$; moreover, it satisfies the "dual" property of (5.10), that is, for every $x \in \mathbb{R}^{N}$ and every $0<r<R$ we have

$$
\left(\frac{R}{r}\right)^{\frac{1}{Q-2}} H(x, r) \leq H(x, R) \leq\left(\frac{R}{r}\right)^{\frac{1}{N-2}} H(x, r) .
$$

By means of such a function (and of Theorem [5.3), we can write a precise relation between $\Gamma$-balls and $d x$-balls: in fact, since $\gamma(x, y)=1 / \Gamma(x, y)$ can be estimated (from above and from below) by $E(x, d x(x, y)$ ), we have (see Remark 3.3)

$$
B x\left(x, H\left(x, r / c_{2}\right)\right) \subseteq \Omega(x, r) \subseteq B x\left(x, H\left(x, c_{2} r\right)\right)
$$

for every $x \in \mathbb{R}^{N}$ and every $r>0$ (here, $c_{2}$ is the constant in Theorem 5.3). As a consequence of this identity, we easily obtain the following lemma.

Lemma 5.6. There exists an absolute constant $c_{3} \geq 1$ such that

$$
\frac{1}{c_{3}}\left(r H^{2}(x, r)\right) \leq|\Omega(x, r)| \leq c_{3}\left(r H^{2}(x, r)\right)
$$

for every $x \in \mathbb{R}^{N}$ and every $r>0$.

Proof. Let $x \in \mathbb{R}^{N}$ be fixed and let $r>0$. By the above (5.18) and Theorem 5.1, we have (see also (5.8) and remind that $H(x, \cdot)$ is the inverse of $E(x, \cdot)$ )

$$
\begin{aligned}
|\Omega(x, r)| & \geq\left|B x\left(x, H\left(x, r / c_{2}\right)\right)\right| \stackrel{\sqrt[5.17]{\geq}}{\geq} \frac{1}{c_{1}} \Lambda\left(x, H\left(x, r / c_{2}\right)\right) \\
& \stackrel{(5.8)}{=} \frac{1}{c_{1}} E\left(x, H\left(x, r / c_{2}\right)\right) \cdot H^{2}\left(x, r / c_{2}\right) \\
& =\frac{1}{c_{1} c_{2}}\left(r H^{2}\left(x, r / c_{2}\right)\right)=(\star)
\end{aligned}
$$

From this, by the second inequality in (5.17), we obtain (remind that $c_{2} \geq 1$ )

$$
(\star) \geq \frac{1}{c_{1} c_{2}} c_{2}^{2 /(N-2)}\left(r H^{2}(x, r)\right)=\frac{1}{c_{3}}\left(r H^{2}(x, r)\right) .
$$

The second inequality in (5.19) can be demonstrated analogously. 
We can now prove that $\mathcal{L}$ satisfies (4.2).

Proposition 5.7. There exists an absolute constant $c_{4} \geq 1$ such that

$$
\frac{1}{c_{4}}\left(\frac{R}{r}\right)^{\frac{Q}{Q-2}}|\Omega(x, r)| \leq|\Omega(x, R)| \leq c_{4}\left(\frac{R}{r}\right)^{\frac{N}{N-2}}|\Omega(x, r)|
$$

for every $x \in \mathbb{R}^{N}$ and every $R, r \in(0, \infty)$ with $r<R$.

Proof. Let $x \in \mathbb{R}^{N}$ be fixed and let $r, R \in(0, \infty)$ be such that $r<R$. By combining Lemma 5.6 with the first inequality in (5.17), we obtain

$$
\begin{aligned}
|\Omega(x, R)| & \geq \frac{1}{c_{3}}\left(R H^{2}(x, R)\right) \stackrel{\sqrt[5.17]{\geq}}{\geq} \frac{1}{c_{3}}\left(\frac{R}{r}\right)^{2 /(Q-2)}\left(R H^{2}(x, r)\right) \\
& =\frac{1}{c_{3}}\left(\frac{R}{r}\right)^{Q /(Q-2)}\left(r H^{2}(x, r)\right) \\
& \frac{(5.19)}{\geq} \frac{1}{c_{3}^{2}}\left(\frac{R}{r}\right)^{Q /(Q-2)}|\Omega(x, r)| .
\end{aligned}
$$

The second inequality in (5.20) can be proved analogously.

Gathering together all the facts proved in these paragraphs we obtain the following result, which is a restatement of Theorem 1.9 in the present setting.

Theorem 5.8. Let $X_{1}, \ldots, X_{m}$ be linearly independent smooth vector fields on $\mathbb{R}^{N}$ (with $N \geq 3$ ) satisfying the assumptions (I)-(II) introduced in the Introduction. Moreover, let $\mathcal{L}=\sum_{j=1}^{m} X_{j}^{2}$. Then, the following facts hold true:

(1) An open set $\Omega \subseteq \mathbb{R}^{N}$ is a maximum principle set for $\mathcal{L}$ if and only if its complement $\mathbb{R}^{N} \backslash \Omega$ is L-large at infinity.

(2) If $\Omega \subseteq \mathbb{R}^{N}$ is an open set such that its complement $\mathbb{R}^{N} \backslash \Omega$ is $p_{\mathcal{L}}$-unbounded (for some $p>1$ ), then $\Omega$ is a maximum principle set for $\mathcal{L}$.

(3) If $\Omega \subseteq \mathbb{R}^{N}$ is an open set such that its complement $\mathbb{R}^{N} \backslash \Omega$ contains a $\Gamma$-cone, then $\Omega$ is a maximum principle set for $\mathcal{L}$.

We now proceed in this section by proving Proposition 1.12 stated in the Introduction. To this end, we first establish the following result.

Proposition 5.9. Let $F \subseteq \mathbb{R}^{N}$ be a non-degenerate $\delta_{\lambda}$-cone, according to Definition 1.11. Then $F$ is a $\Gamma$-cone.

Proof. According to Definition 1.7, we have to prove the existence of a countable family $\mathcal{F}=\left\{\Omega\left(z_{n}, R_{n}\right)\right\}_{n}$ such that $\Omega\left(z_{n}, R_{n}\right) \subseteq F$ for any $n \in \mathbb{N}$ and

(a) $\left\|z_{n}\right\| \rightarrow \infty$ as $n \rightarrow \infty$;

(b) $\liminf _{n \rightarrow \infty} R_{n} / \gamma_{0}\left(z_{n}\right)>0$.

To this end, we fix $z_{0} \in \operatorname{int}(F) \backslash\{0\}$ and we let $R_{0}>0$ be such that $\Omega\left(z_{0}, R_{0}\right) \subseteq F$. Chosen a sequence $\left\{\lambda_{n}\right\}_{n} \subseteq\left(\lambda_{0}, \infty\right)$ diverging to $\infty$ as $n \rightarrow \infty$, we define

$$
\Omega_{n}=\Omega\left(z_{n}, R_{n}\right):=\Omega\left(\delta_{\lambda_{n}}\left(z_{0}\right), \lambda_{n}^{Q-2} R_{0}\right) \quad \text { for every } n \in \mathbb{N} .
$$


Since the fundamental solution $\Gamma$ of $\mathcal{L}$ is jointly homogeneous of degree $2-Q$, it is straightforward to recognize that, for every $n \in \mathbb{N}$,

$$
\Omega_{n}=\delta_{\lambda_{n}}\left(\Omega\left(z_{0}, R_{0}\right)\right)
$$

hence, by property (ii) of $F$ we have $\Omega_{n} \subseteq F$ for any $n \in \mathbb{N}$. Furthermore, we have $\left\|z_{n}\right\|=\left\|\delta_{\lambda_{n}}\left(z_{0}\right)\right\| \rightarrow \infty$ as $n \rightarrow \infty$ and, again by jointly homogeneity of $\Gamma$,

$$
R_{n} / \gamma_{0}\left(z_{n}\right)=R_{0} / \gamma_{0}\left(z_{0}\right)>0, \quad \text { for every } n \in \mathbb{N} \text {. }
$$

This shows that $\mathcal{F}:=\left\{\Omega_{n}\right\}_{n}$ is a countable family of $\Gamma$-balls (contained in $F$ ) satisfying (a) and (b), whence $F$ is a $\Gamma$-cone.

Remark 5.10. Let $v \in \mathbb{R}^{N} \backslash\{0\}$ be fixed and let $h \in \mathbb{R}$. Then the half-space

$$
\Pi:=\left\{x \in \mathbb{R}^{N}:\langle x, v\rangle \geq h\right\}
$$

contains a $\delta_{\lambda}$-cone. Indeed, if we consider the subset of $\Pi$ defined by

$$
C:=\left\{x \in \Pi: x_{i} v_{i} \geq 0 \text { for any } i=1, \ldots, N\right\}
$$

it is very easy to recognize that $\operatorname{int}(C) \neq \varnothing$; moreover, $\delta_{\lambda}(x) \in C$ for every $x \in C$ and every $\lambda>1$. Hence, $C$ is a (non-degenerate) $\delta_{\lambda}$-cone contained in $\Pi$.

By combining the above Proposition [5.9] with Theorem [1.8, we are able to provide a very simple proof of Proposition 1.12

Proof (of Proposition 1.12). Let $F \subseteq \mathbb{R}^{N}$ be as in the statement of the proposition. By assumption, there exists a non-degenerate $\delta_{\lambda}$-cone $C \subseteq F$; on the other hand, by Proposition 5.9, $C$ is a $\Gamma$-cone (in the sense of Definition 1.7); as a consequence, from Theorem 1.8 we infer the existence of a suitable $p>1$ such that $F$ is $p_{\mathcal{L}}$-unbounded. This ends the proof.

The next Proposition [5.11] which is the last result of the section, contains a useful characterization of the notion of $p_{\mathcal{L}}$-boundedness in terms of the control distance $d_{\mathrm{X}}$ (associated with the vector fields $\left.X_{1}, \ldots, X_{m}\right)$.

Proposition 5.11. Let $F \subseteq \mathbb{R}^{N}$ be any (non-void) set and let $p \in(1, \infty)$. Then, $F$ is $p_{\mathcal{L}}$-bounded if and only if $F$ satisfies the following property: there exists countable family $\mathcal{G}=\left\{B_{\mathrm{X}}\left(x_{n}, \rho_{n}\right)\right\}_{n \in J}$ such that (setting $d_{\mathrm{X}}(x):=d_{\mathrm{X}}(0, x)$ )

$$
(\diamond) \quad F \subseteq \bigcup_{n \in J} B_{\mathrm{X}}\left(x_{n}, \rho_{n}\right) \quad \text { and } \quad \sum_{n \in J}\left(\frac{E\left(x_{n}, \rho_{n}\right)}{d_{X}^{Q-2}\left(x_{n}\right)}\right)^{p}<\infty
$$

Proof. $(\Rightarrow)$ Since, by assumption, $F$ is $p_{\mathcal{L}}$-bounded, it is possible to find a countable family $\mathcal{F}=\left\{\Omega\left(x_{n}, r_{n}\right)\right\}_{n \in J}$ such that (see Definition 1.4)

$$
F \subseteq \bigcup_{n \in J} \Omega\left(x_{n}, r_{n}\right) \quad \text { and } \quad \sum_{n \in J}\left(\Gamma_{0}\left(x_{n}\right) r_{n}\right)^{p}<\infty
$$

on the other hand, by the second inclusion in (5.18), for every $n \in J$ we have

$$
\Omega\left(x_{n}, r_{n}\right) \subseteq B_{X}\left(x_{n}, \rho_{n}\right), \quad \text { where } \rho_{n}=H\left(x_{n}, c_{2} r_{n}\right) .
$$


Thus, if we consider the family $\mathcal{G}=\left\{B_{\mathrm{X}}\left(x_{n}, \rho_{n}\right)\right\}_{n \in J}$, we see that $\mathcal{G}$ is a countable cover of $F$ such that (remind that $H(x, \cdot)=(E(x, \cdot))^{-1}$ )

$$
\infty>\sum_{n \in J}\left(\Gamma_{0}\left(x_{n}\right) r_{n}\right)^{p}=\frac{1}{c_{2}^{p}} \sum_{n \in J}\left(\Gamma_{0}\left(x_{n}\right) E\left(x_{n}, \rho_{n}\right)\right)^{p}=:(\star) .
$$

We now turn to give an estimate of $\Gamma_{0}\left(x_{n}\right)$ in terms of $d_{x}\left(x_{n}\right)$. To this end we observe that, by the first inequality in (5.14), we have

$$
\Gamma_{0}\left(x_{n}\right) \geq \frac{1}{c_{2}} \frac{d_{x}^{2}\left(x_{n}\right)}{\Lambda\left(0, d_{x}\left(x_{n}\right)\right)} \quad \text { for every } n \in J
$$

from this, taking into account (5.12) in Remark 5.2- (d) $)_{1}$, we obtain

$$
\Gamma_{0}\left(x_{n}\right) \geq \frac{1}{\omega_{Q} c_{2}} \cdot \frac{1}{d_{\mathrm{X}}^{Q-2}\left(x_{n}\right)} \quad \text { for every } n \in J .
$$

By means of this last estimate we conclude that

$$
\infty>(\star) \geq\left(\frac{1}{\omega_{Q} c_{2}^{2}}\right)^{p} \sum_{n \in J}\left(\frac{E\left(x_{n}, \rho_{n}\right)}{d_{X}^{Q-2}\left(x_{n}\right)}\right)^{p},
$$

and this proves that $\mathcal{G}$ satisfies $(\diamond)$.

$(\Leftarrow)$ Let $\mathcal{G}=\left\{B_{x}\left(x_{n}, \rho_{n}\right)\right\}_{n \in J}$ be a countable family of $d x$-balls satisfying $(\diamond)$. By the first inclusion in (5.18) (and again by the fact that the maps $E(x, \cdot)$ are $H(x, \cdot)$ are inverse to each other), it is easy to recognize that

$$
B x\left(x_{n}, \rho_{n}\right) \subseteq \Omega\left(x_{n}, r_{n}\right), \quad \text { where } r_{n}=c_{2} \cdot E\left(x_{n}, \rho_{n}\right) ;
$$

thus, if we consider the family $\mathcal{F}:=\left\{\Omega\left(x_{n}, r_{n}\right)\right\}_{n \in J}$, we see that $\mathcal{F}$ is a countable cover of $F$ (since the same is true of $\mathcal{G}$ ) and that

$$
\infty>\sum_{n \in J}\left(\frac{E\left(x_{n}, \rho_{n}\right)}{d_{X}^{Q-2}\left(x_{n}\right)}\right)^{p}=\frac{1}{c_{2}^{p}} \sum_{n \in J}\left(\frac{r_{n}}{d_{X}^{Q-2}\left(x_{n}\right)}\right)^{p}=:(\star) .
$$

On the other hand, by using the second inequality in (5.14) and by using again (5.12) in Remark 5.2- $(\mathrm{d})_{1}$, we derive that

$$
\Gamma_{0}\left(x_{n}\right) \leq c_{2} \frac{d_{X}^{2}\left(x_{n}\right)}{\Lambda\left(0, d_{x}\left(x_{n}\right)\right)}=\frac{c_{2}}{\omega_{Q}} \cdot \frac{1}{d^{Q-2}\left(x_{n}\right)} \quad \text { for every } n \in J
$$

as a consequence, we obtain

$$
\infty>(\star) \geq\left(\frac{\omega_{Q}}{c_{2}^{2}}\right)^{p} \sum_{n \in J}\left(\Gamma_{0}\left(x_{n}\right) r_{n}\right)^{p}
$$

and this proves that $F$ is $p_{\mathcal{L}}$-bounded.

Remark 5.12. As a final remark we observe that, in the particular case when $X_{1}, \ldots, X_{m}$ are Lie generators of the Lie algebra of some homogeneous Carnot group on $\mathbb{R}^{N}$ (see [13, Chapter 1] for the relevant definitions), we have

$$
E(x, r)=\omega_{Q} r^{Q-2} \quad \text { for every } x \in \mathbb{R}^{N} \text { and every } r>0 ;
$$


as a consequence, a set $F \subseteq \mathbb{R}^{N}$ is $p_{\mathcal{L}}$-bounded (for some $p>1$ ) if and only if there exists a countable family $\mathcal{G}=\left\{B_{\mathrm{X}}\left(x_{n}, \rho_{n}\right)\right\}_{n \in J}$ such that

$$
F \subseteq \bigcup_{n \in J} B_{\mathrm{X}}\left(x_{n}, \rho_{n}\right) \quad \text { and } \quad \sum_{n \in J}\left(\frac{\rho_{n}}{d_{X}\left(x_{n}\right)}\right)^{p(Q-2)}<\infty .
$$

Due to this fact, the results presented in this paper comprehend and generalize that contained in [12] (see also [13, Chapter 10]).

\section{A Appendix: some results of Potential Theory}

The main aim of this brief appendix is to collect some notions and results, coming from Potential Theory, needed to prove Lemma 2.1 in Section 2 . In our exposition we mainly follow the book by Brelot 15 , to which we refer for a detailed treatment of these topics (and for the proof of all the results we are going to state); we also highlight the very classical references [1, 16].

Throughout the sequel, we denote by $\mathcal{L}$ a fixed linear PDO as in (1.1) and satisfying the structural assumptions (H1)-to-(H3); moreover, we tacitly inherit all the notations introduced in the previous sections.

\section{The L-harmonic space}

We begin with the following simple observation: if $\tau_{\mathcal{E}}$ denotes the usual Euclidean topology on $\mathbb{R}^{N}$, then the assignment

$$
\tau_{\mathcal{E}} \ni \Omega \mapsto \mathcal{L}(\Omega)=\left\{u \in C^{2}(\Omega, \mathbb{R}): \mathcal{L} u=0 \text { in } \Omega\right\},
$$

is a sheaf of functions on $\mathbb{R}^{N}$. More precisely, we have

(i) for any $\Omega \in \tau_{\mathcal{E}}, \mathcal{L}(\Omega)$ is a linear subspace of $C(\Omega, \mathbb{R})$;

(ii) if $\Omega_{1} \subseteq \Omega_{2}$ are open subsets of $\mathbb{R}^{N}$ and if $u \in \mathcal{L}\left(\Omega_{2}\right)$, then $u_{\Omega_{1}} \in \mathcal{L}\left(\Omega_{1}\right)$;

(iii) if $\left\{\Omega_{i}\right\}_{i \in I} \subseteq \tau_{\mathcal{E}}$ and if $u: \Omega:=\bigcup_{i \in I} \Omega_{i} \rightarrow \mathbb{R}$, then

$$
\left(u_{\left.\right|_{i}} \in \mathcal{L}\left(\Omega_{i}\right) \text { for all } i \in I\right) \Longrightarrow u \in \mathcal{L}(\Omega) .
$$

Let now $\Omega \subseteq \mathbb{R}^{N}$ be an open set. We say that $\Omega$ is $\mathcal{L}$-regular if

(i) $\bar{\Omega}$ is compact;

(ii) for every continuous function $f: \partial \Omega \rightarrow \mathbb{R}$ there exists a unique $\mathcal{L}$-harmonic function in $\Omega$, denoted by $H_{f}^{\Omega}$, such that

$$
\lim _{x \rightarrow \xi} H_{f}^{\Omega}(x)=f(\xi), \quad \text { for all } \xi \in \partial \Omega
$$

(iii) if $f \geq 0$ on $\partial \Omega$, then $H_{f}^{V} \geq 0$ in $\Omega$. 
It is very easy to see that, if $\Omega$ is $\mathcal{L}$-regular, the map

$$
T: C(\partial \Omega, \mathbb{R}) \longrightarrow \mathbb{R}, \quad T(f):=H_{f}^{\Omega}(x)
$$

is linear and positive; since $\partial \Omega$ is compact, the Riesz Representation Theorem (see, e.g., [21]) provides us with a unique Radon measure $\mu_{x}^{\Omega}$ on $\Omega$ such that

$$
H_{f}^{\Omega}(x)=\int_{\partial \Omega} f(y) \mathrm{d} \mu_{x}^{\Omega}(y)
$$

The measure $\mu_{x}^{\Omega}$ is called the $\mathcal{L}$-harmonic measure related to $\Omega$ and $x$.

As a consequence of some results proved in [4] (see, precisely, Lemma 1.7 and Theorem 1.10), we see that the following facts hold true for our PDO $\mathcal{L}$ :

(a) there exists a (countable) basis for the Euclidean topology of $\mathbb{R}^{N}$ consisting of connected $\mathcal{L}$-regular open sets;

(b) for every connected open set $\Omega \subseteq \mathbb{R}^{N}$ and every compact set $K \subseteq \Omega$, there exists a constant $C=C(\Omega, K) \geq 1$ such that

$$
\sup _{K} u \leq C \inf _{K} u,
$$

for every non-negative harmonic function $u$ in $\Omega$.

On the other hand, the validity of (a) and (b) easily implies the following results.

(1) Let $\Omega \subseteq \mathbb{R}^{N}$ be an open set (not necessarily $\mathcal{L}$-regular) and let $u \in C(\Omega, \mathbb{R}$ ). Then the function $u$ is $\mathcal{L}$-harmonic in $\Omega$ if and only if

$$
u(x)=\int_{\partial V} u(z) \mathrm{d} \mu_{x}^{V}(z),
$$

for every $\mathcal{L}$-regular open set $V \subseteq \bar{V} \subseteq \Omega$ and every $x \in V$.

(2) If $\Omega \subseteq \mathbb{R}^{N}$ is open and connected and $\left\{u_{n}\right\}_{n} \subseteq \mathcal{L}(\Omega)$ is monotone increasing, then either $\sup _{n} u_{n} \equiv \infty$ on $\Omega$ or it is a $\mathcal{L}$-harmonic function in $\Omega$.

Gathering together all these facts, we recognize that the map defined in (A.1) satisfies Axioms 1-to-3 in [15]; hence, it endows $\mathbb{R}^{N}$ with the structure of a harmonic sheaf, which is usually referred to as the $\mathcal{L}$-harmonic space.

\section{$\mathcal{L}$-subharmonic functions}

Let $\Omega \subseteq \mathbb{R}^{N}$ be an open set and let $u: \Omega \rightarrow[-\infty, \infty)$ be u.s.c. on $\Omega$. As already said in the Introduction, the function $u$ is $\mathcal{L}$-subharmonic in $\Omega$ if

(i) $\{x \in \Omega: u(x)>-\infty\}$ is dense in $\Omega$;

(ii) for every bounded open set $V \subseteq \bar{V} \subseteq \Omega$ and for every function $h \mathcal{L}$-harmonic in $V$ and continuous up to $\partial V$ such that $u_{\left.\right|_{\partial V}} \leq h_{\left.\right|_{\partial V}}$, one has $u \leq h$ in $V$. 
Remark A.1. Let $\Omega \subseteq \mathbb{R}^{N}$ be open and let $u \in \underline{L}(\Omega)$. Moreover, let $V$ be a $\mathcal{L}$-regular open set such that $\bar{V} \subseteq \Omega$. If $\varphi \in C(\partial V, \mathbb{R})$ is any continuous function satisfying $u \leq \varphi$ on $\partial V$, by (ii) we have

$$
u(x) \leq H_{\varphi}^{V}(x)=\int_{\partial V} \varphi(z) \mathrm{d} \mu_{x}^{V}(z) \text { for every } x \in V .
$$

From this, due to the arbitrariness of $\varphi$, we obtain

$$
u(x) \leq \int_{\partial V} u(z) \mathrm{d} \mu_{x}^{V}(z) \text { for every } x \in V .
$$

For $\mathcal{L}$-subharmonic functions, we have the following minimum principles.

Theorem A.2. Let $\Omega \subseteq \mathbb{R}^{N}$ be open and let $u \in \underline{\mathcal{L}}(\Omega)$. The following facts hold:

1. if $\Omega$ is connected and $u \leq 0$ on $\Omega$, then either $u \equiv 0$ or $u<0$;

2. if $\Omega$ is bounded, $\liminf _{x \rightarrow \xi} u(x) \leq 0$ for any $\xi \in \partial \Omega$ and there exists $a$ $\mathcal{L}$-harmonic function $h$ such that $\inf _{\Omega} h>0$, then $u \leq 0$ on $\Omega$.

Theorem A.2 allows us to prove that condition (A.2) in Remark A.1 actually characterizes, even in a suitable local form, $\mathcal{L}$-subharmonicity.

Proposition A.3. Let $\Omega \subseteq \mathbb{R}^{N}$ be open and let $u: \Omega \rightarrow(-\infty, \infty]$ be a u.s.c. function such that the set $D:=\{x \in \Omega: u(x)>-\infty\}$ is dense in $\Omega$.

Then the following conditions are equivalent:

(a) $u \in \underline{\mathcal{L}}(\Omega)$;

(b) for every $\mathcal{L}$-regular open set $V \subseteq \bar{V} \subseteq \Omega$ and for every function $\varphi \in C(\partial V, \mathbb{R})$ satisfying $u \leq \varphi$ on $\partial V$, one has

$$
u(x) \leq \int_{\partial V} u(z) \mathrm{d} \mu_{x}^{V}(z) \quad \text { for every } x \in V .
$$

(c) for every $x_{0} \in \Omega$ there exists a basis $\mathcal{B}_{u}$ (possibly depending on $u$ ) of $\mathcal{L}$ regular open neighborhoods of $x_{0}$ such that, for any $V \in \mathcal{B}_{u}$, one has

$$
u\left(x_{0}\right) \leq \int_{\partial V} u(y) \mathrm{d} \mu_{x_{0}}^{V}(y) .
$$

\section{Proof of Lemma 2.1}

Thanks to Proposition A.3. we are finally in a position to prove Lemma 2.1. For the sake of clarity, we re-write here its statement.

Lemma A.4. Let $\Omega \subseteq \mathbb{R}^{N}$ be open and let $u \in \underline{\mathcal{L}}(\Omega)$ be such that

$$
\limsup _{x \rightarrow \xi} u(x) \leq 0 \quad \text { for every } \xi \in \partial \Omega .
$$

Then the function $v: \mathbb{R}^{N} \rightarrow[-\infty, \infty)$ defined by

$$
v(x)= \begin{cases}\max \{u(x), 0\}, & \text { if } x \in \Omega, \\ 0, & \text { if } x \in \mathbb{R}^{N} \backslash \Omega,\end{cases}
$$

is $\mathcal{L}$-subharmonic in $\mathbb{R}^{N}$. 
Proof. First of all, condition (A.3) ensures that $v$ is u.s.c. on $\Omega$; moreover, by the very definition of $v$, we have $v \geq 0>-\infty$ on the whole of $\mathbb{R}^{N}$.

To prove that $v \in \underline{L}\left(\mathbb{R}^{N}\right)$ we show that, for every $x_{0} \in \mathbb{R}^{N}$, there exists a basis $\mathcal{B}\left(x_{0}\right)$ of $\mathcal{L}$-regular open neighborhoods of $x_{0}$ such that (see Proposition A.3)

$$
v\left(x_{0}\right) \leq \int_{\partial V} v(y) \mathrm{d} \mu_{x_{0}}^{V}(y), \quad \text { for every } V \in \mathcal{B}\left(x_{0}\right) .
$$

If $x_{0} \in \Omega$, we can choose as $\mathcal{B}\left(x_{0}\right)$ the family of the $\mathcal{L}$-regular open neighborhoods of $x_{0}$ with closure contained in $\Omega$ : indeed, since $f:=\max \{u, 0\}$ is $\mathcal{L}$-subharmonic in $\Omega$ (as the same is true of both $u$ and 0 ), we have

$$
v\left(x_{0}\right)=f\left(x_{0}\right) \leq \int_{\partial V} f(y) \mathrm{d} \mu_{x_{0}}^{V}(y)=\int_{\partial V} u(y) \mathrm{d} \mu_{x_{0}}^{V}(y)
$$

for every $\mathcal{L}$-regular open neighborhood $V$ of $x_{0}$ with $\bar{V} \subseteq \Omega$. If, instead, $x_{0} \notin \Omega$, we can choose as $\mathcal{B}\left(x_{0}\right)$ the family of all $\mathcal{L}$-regular open neighborhoods of $x_{0}$ : indeed, since $v \geq 0$ on the whole of $\mathbb{R}^{N}$ (by definition), we have

$$
v\left(x_{0}\right)=0 \leq \int_{\partial V} u(y) \mathrm{d} \mu_{x_{0}}^{V}(y)
$$

for every $\mathcal{L}$-regular open neighborhood $V$ of $x_{0}$. This ends the proof.

\section{References}

[1] H. Bauer: Harmonische Räume und ihre Potentialtheorie, Lecture Notes in Mathematics 22, Springer-Verlag, Berlin-New York, 1966.

[2] E. Battaglia, A. Bonfiglioli: Normal families of functions for subelliptic operators and the theorems of Montel and Koebe, J. Math. Anal. Appl. 409, 1-12 (2014).

[3] E. Battaglia, S. Biagi: Superharmonic functions associated with hypoelliptic non-Hörmander operators, Comm. Cont. Math. (2018). doi: 10.1142/S0219199718500712

[4] E. Battaglia, S. Biagi, A. Bonfiglioli: The strong maximum principle and the Harnack inequality for a class of hypoelliptic non-Hörmander operators, Ann. Inst. Fourier (Grenoble) 66, 589-631 (2016).

[5] H. Berestycki, L. Nirenberg: On the method of moving planes and the sliding method, Boletim Soc. Brasil. de Mat. Nova Ser. 22, 1-37 (1991).

[6] H. Berestycki, L. Caffarelli, L. Nirenberg: Monotonicity for elliptic equations in unbounded Lipschitz domains, Comm. Pure Appl. Math. 50, 1089-1111 (1997).

[7] H. Berestycki, F. Hamel, R. Monneau: One-dimensional symmetry of bounded entire solutions of some elliptic equations, Duke Math. J. 103, 375-396 (2000). 
[8] S. Biagi, A. Bonfiglioli: The existence of a global fundamental solution for homogeneous Hörmander operators via a global Lifting method, Proc. London Math. Soc. 114, 855-889 (2017).

[9] S. Biagi, A. Bonfiglioli and M. Bramanti: Global estimates for the fundamental solution of homogeneous Hörmander sums of squares submitted, arXiv:1906.07836 (2019).

[10] I. Birindelli, E. Lanconelli: A negative answer to a one-dimensional symmetry problem in the Heisenberg group, Calc. Var. Partial Differential Equations 18, 357-372 (2002).

[11] I. Birindelli, J. Prajapat: One dimensional symmetry in the Heisenberg group, Ann. Scuola Norm. Sup. Pisa Cl. Sci. 30, 269-284 (2001).

[12] A. Bonfiglioli, E. Lanconelli: Maximum Principle on Unbounded Domains for sub-Laplacians: a Potential Theory approach, Proc. Amer. Math. Soc. 130, 2295-2304 (2002).

[13] A. Bonfiglioli, E. Lanconelli, F. Uguzzoni: Stratified Lie Groups and Potential Theory for their sub-Laplacians, Springer Monographs in Mathematics 26, Springer, New York, N.Y., 2007.

[14] M. Bramanti: An Invitation to Hypoelliptic Operators and Hörmander's Vector Fields SpringerBriefs in Mathematics, Springer, New York, N.Y., 2014.

[15] M. Brelot: Axiomatique des fonctions harmoniques, Les Presses de l'Université de Montréal, Montréal, 1969.

[16] C. Constantinescu, A. Cornea: Potential theory on Harmonic Spaces, Springer-Verlag, 1972.

[17] W.K. Hayman, P.B. Kennedy: Sub-Harmonic Functions, Volume I, Academic Press, London (1976).

[18] A.E. Kogoj, E. Lanconelli: Liouville theorems for a class of linear secondorder operators with nonnegative characteristic form, Bound. Value Probl. (2007).

[19] A.E. Kogoj, E. Lanconelli: On semilinear $\Delta_{\lambda}$-Laplace equations, Nonlinear Anal. 75, 4637-4649 (2012).

[20] A. Nagel, E.M. Stein, S. Wainger: Balls and metrics defined by vector fields I, basic properties, Acta Math. 155, 103-147 (1985).

[21] W. Rudin: "Real and Complex Analysis", McGraw-Hill, New York, 1987. 